\title{
Information Environments and High Price Impact Trades: Implication for
}

\section{Volatility and Price Efficiency*}

\section{November 2019}

\author{
Georges Dionne $^{a, b} \quad$ Xiaozhou Zhou ${ }^{a, c \dagger}$ \\ ${ }^{a}$ Canada Research Chair in Risk Management, HEC Montréal, Montréal H3T 2A7, Canada \\ ${ }^{b}$ Finance Department, HEC Montréal, Montréal H3T 2A7, Canada \\ ${ }^{c}$ Faculty of Management (ESG), University of Quebec at Montreal, H3C 3P8, Canada
}

\begin{abstract}
We include trade matchedness to Limit Order Book (LOB) as an extended identification dimension of High Price Impact Trades (HPITs). HPITs are trades associated with disproportionately large price changes in comparison with their volume proportion and are related to informed trades. We show that the introduction of matchedness provides finer informed trading identification, and we find that a stronger presence of HPITs leads to a decline in volatility due to more contrarian informed trades, but this decline varies with information environments. Finally, we conclude that HPITs mainly reflect belief-based (fundamentalbased) information in a high (low) public disclosure environment.
\end{abstract}

Keywords: Price efficiency, Price discovery, Limit Order Book, Trade size clustering, Stealth trading.

JEL classification: C22 C41 C53 G11

${ }^{*}$ We thank Yann Bilodeau for his help in constructing the dataset and comments. We also thank Antonia Kirilova, Vineet Virmani, Jun Wen, and participants at the 2019 Cross Country Perspective in Finance Symposium, 2019 China Conference of CEA(UK/Europe) and the 31th Asian FA Annual Meeting for their remarks.

${ }^{\dagger}$ Corresponding author at: Faculty of Management (ESG), University of Quebec at Montreal (UQAM), 315 rue Sainte-Catherine Est Montreal, Quebec, Canada, H2X 3X2. Tel.: +1 5149873000 ext 0999. E-mail addresses: georges.dionne@hec.ca (G. Dionne), zhou.xiaozhou@uqam.ca (X. Zhou). 


\section{Introduction}

Financial markets feature two important types of informed traders: one possesses private information about companies' fundamental value, which is not yet incorporated in the current price, and the other has information about who might have such fundamental-related information. Regardless of the nature and source of information, both types of informed (information-related) investors attempt to maximize their profits by trading on their superior information. The resulted trading behaviors correspond to fundamental-based and belief-based trades in the literature, ${ }^{1}$ and imply two important reinforcing channels that facilitate the price discovery process, either by making the security price reflect new private information or by correcting the price distortion caused by uninformed liquidity shocks. Obvious examples include fundamental informed traders, who buy or sell the corresponding stocks according to the potential impact of their value-relevant private information, and financial intermediaries, who correct the price deviation caused by identified uninformed liquidity traders or trade in the same direction as identified informed traders. ${ }^{2}$ In real financial markets, there also exist uninformed liquidity traders (institutional or retail), ${ }^{3}$

\footnotetext{
${ }^{1}$ Throughout the paper, we use information-related trades or informed trades to refer to the trades derived from fundamental-based and belief-based traders. Specifically, our definition of fundamental-based traders is in the spirit of the study by Goldstein and Yang (2015), which supposes that people are heterogeneously informed about one dimension of fundamental values. Also, we classify activists' information as fundamental-related information even though the fundamental value changes when activists purchase a significant number of shares. By definition, activists are outside shareholders who identify a firm for potential value creation through their effort expenditure (Collin-Dufresne and Fos (2015)). Before activists publicly announce their intention to influence corporate governance, they attempt to purchase a significant number of shares in the open market. In contrast, belief-based traders differ from fundamental-based traders by the information they use. Typically, they exhibit reactive trading behavior that acts on information about trades by other traders. Belief-based traders profit when they correctly identify the trading motivation of other investors.

${ }^{2}$ Our financial intermediaries might be traditional or sophisticated high-frequency market makers who have access to both public information and information related to their customers. The migration to the electronic order-driven market and the use of high-performance computers led to the rise of high-frequency trading and market making in financial markets. Today, sophisticated market making algorithms can quickly learn about other traders' trading motivations by following the footprints they leave in the market. For example, Korajczyk and Murphy (2018) and Van Kervel and Menkveld (2019) show that it takes some time for high frequency market makers to detect fundamental-based trades. Typically, they initially lean against informed traders, but ultimately they are able to identify the most informed trading and trade with the wind. In addition, both traditional and high frequency market makers can exploit the information related to their customers. Having their customers' whole trading history, market makers possess at least superior private information about the performance of their customers and are in fact well positioned to judge if someone is informed or not. Also, in many cases, they possess information about other brokers. Even though individual brokers cannot identify the exact identity of the traders behind other brokers, they still have a nonfundamental informational advantage. Therefore, belief-based (non-fundamental) information will let market makers better assess the likelihood that the ongoing trades are derived from un(informed) traders than any uninformed liquidity traders. Note that, in practice, market makers were not allowed to trade on the information derived from their clients, but enforcement was extremely difficult.

${ }^{3}$ In the market microstructure literature, these uninformed trades are defined as discretionary liquidity trades, and have been studied by Admati and Pfleiderer (1988) and Foster (1990). We follow the literature and use the terms "noise trading", "liquidity trading", and "uninformed trading" interchangeably.
} 
which trade for exogenous discretionary reasons, and decide on which financial market to participate in according to liquidity costs and availability of public information. Together, informed traders (fundamental-based and belief-based) and uninformed traders constitute the financial markets. The interaction between them creates the dynamics of short-term security prices. How to accurately identify informed trades and recognize the type of information conveyed by such informed trades, along with their implications for market quality, remain important open questions in the empirical finance literature.

To address these questions, we integrate the literature on identification of informed trading, endogenous information acquisition in the financial market, and the impact of informed trading on market quality in different information environments. ${ }^{4}$ Our study is carried out in three steps. First, we take the information embedded in the LOB as a new dimension to identify high price impact trades, and relate them to informed trades. Second, we identify the type of information conveyed by these high impact trades by empirically evaluating their effect on shortterm volatility and return autocorrelation for the stocks in different information environments. Third, we provide evidence that, in various information environments, the implications of high price impact trades for price efficiency differ.

Our empirical study uses stocks from three indexes of the Deutsche Börse: DAX, MDAX, and SDAX, which are traded at the Frankfurt Stock Exchange (FSE). The distinction between DAX, MDAX, and SDAX provides a natural setting in terms of information environments to study the role or impact of information-related trades on intraday price formation, volatility and price efficiency. Specifically, the DAX index is composed of the 30 largest German companies in terms of market capitalization and Limit Order Book volume (LOB liquidity), which is widely considered as a blue chip market index for the German stock market. In addition, DAX stocks have a large number of analysts, lower forecast dispersion among analysts and smaller forecast error. ${ }^{5}$ Based on these facts, it is fair to conclude that 1) the stocks in the DAX index are traded in a high public information environment with high liquidity. 2) DAX stocks are attractive for

\footnotetext{
${ }^{4}$ Market quality is usually measured by volatility and price efficiency, which assesses how well the price reflects the information that is relevant to the asset's fundamental value. We use price efficiency, market efficiency and informational efficiency interchangeably.

${ }^{5}$ The detailed calculations of these variables are presented in Section 3 , and the corresponding results are reported in Table 2 .
} 
uninformed liquidity traders because of a lower expected loss of liquidity (Han et al.(2016)). In contrast, the MDAX index is composed of medium-sized companies, which feature a smaller LOB volume, fewer analysts, higher forecast dispersion and larger forecast error. Finally, the SDAX index includes small-sized companies with the smallest LOB volume, the fewest number of analysts, the highest forecast dispersion and the largest forecast error. Compared with large-cap DAX stocks, both MDAX and SDAX stocks are traded in a low public information environment and are less attractive for uninformed liquidity traders because of a higher liquidity cost.

To identify high price impact trades in different information environments, we start by showing the existence of roundedness clustering of trade volumes and matchedness to the quantity standing in the open LOB. Roundedness clustering either in price or trade size has been a well-recognized phenomenon in many markets. Here, we conduct a distinct test of roundedness clustering from a market where the round-lot of a transaction is one unit, in which traders are free to choose their size for both market and limit orders. Our empirical results show that DAX, MDAX, and SDAX stocks feature trade-size clustering on 10, 50, 100 or their multiples. Rounded trades are significantly present in all three indexes and amount to almost $40 \%$ of the total trading volume.

Another important, but hardly explored, dimension to distinguish trades is matchedness. In this study, we define a trade as a matched trade when its trade size matches the exact quantities available in the LOB. ${ }^{6}$ Specifically, matchedness relates to the responsiveness of liquidity demand of informed or uninformed traders with respect to liquidity supply. Specifically, with the development of the trading system from the quote-driven market to the order-driven market, the operations in financial market have become more transparent and faster than ever. Both high frequency informed and uninformed traders can continuously observe the dynamics of the open LOB and time their order submissions. Despite the importance of the LOB in order submission strategy and price formation, the role of LOB-based trades has been largely ignored in the literature. To the best of our knowledge, this is the first research that uses LOB matchedness of trade to distinguish trades, and links matchedness to high frequency trades. Our study provides two insights into matched trades: first, matched trades represent more than $50 \%$ of total trades

\footnotetext{
${ }^{6}$ Because our dataset does not include fully hidden orders and the hidden part of an iceberg order, our matchedness is based on observable limit orders.
} 
for DAX and MDAX stocks and more than $40 \%$ for SDAX stocks. The omission of matchedness in informed trading identification could result in misleading conclusions. For example, our results show that small-matched trades and small-unmatched trades do not contribute to the price discovery process in the same way. The same pattern holds for (un)rounded-matched trades and (un)rounded-unmatched trades regardless of the index, suggesting that informed traders (human or non-human) are wary of market liquidity and carefully monitor the information embedded in the LOB. Second, the information quality of matched trades, measured by the ratio of cumulative price contribution of matched trades to their corresponding proportion of volume, ${ }^{7}$ varies with trade sizes and information environments. Overall, these findings provide evidence that matchedness, as a complement to roundedness, is an important dimension in informed trading identification. For example, matchedness indicates more information in small trades.

We then classify all trades of the stocks in a given index by size, ${ }^{8}$ roundedness, and matchedness, and statistically identify the trade types that have a high price impact. ${ }^{9}$ We combine all trade types with a high price impact to construct our high price impact trades (HPITs), which, in general, lead to disproportionately large price discovery ${ }^{10}$ relative to their proportion of volume. Our results show that roundedness and matchedness are important dimensions in HPIT identification, and DAX, MDAX and SDAX stocks do not have the same trade types in their HPITs. In addition, the information quality of HPITs, defined as the ratio of the price discovery of HPITs relative to their proportion of volume, ${ }^{11}$ varies across different stock indexes.

Once HPITs are identified, we further analyze their intraday price contribution dynamics and the corresponding volume (expressed as a percentage of daily trade volumes) for stocks in the three indexes. For DAX stocks, ${ }^{12}$ we show that the contribution to price discovery of HPITs dominates that of non-HPITs during the whole continuous trading session $(73.64 \%$ for HPITs vs.

\footnotetext{
${ }^{7}$ An alternative interpretation of the information quality of matched trades is the average increase in price contribution given a one-percent increase in HPITs.

${ }^{8}$ In this study, trades of each stock are classified as small, medium or large when the corresponding trade sizes are smaller than the 30th percentile, between the 30th and 70th percentiles, and larger than the 70th percentile of its own trade size distribution, respectively.

${ }^{9}$ For a given type of trade, we calculate the cumulative (aggregated) price changes derived from all trades within this type.

${ }^{10}$ Price discovery attributed to HPITs is the price change associated with HPITs expressed as a percentage of daily price change.

${ }^{11}$ Similar to the information quality of matched trades, the information quality of HPITs measures the average increase in price contribution given a one-percent increase in HPITs.

${ }^{12}$ For MDAX and SDAX stocks, we observe similar patterns.
} 
26.36 for non-HPITs), accompanied by a striking difference in volume proportion (16.77\% for HPITs vs. $83.23 \%$ for non-HPITs). More importantly, the beginning-of-the-day (9 a.m.-10 a.m.) contribution of HPITs to the daily price changes is comparable to, but always higher than, that of non-HPITs (20\% vs. 15\%). However, after 10 a.m., $82.64 \%$ of total price discovery is made by HPITs. In addition, the intraday volume from non-HPITs features strong seasonality, while the intraday volume from HPITs is quite stable. This observation confirms our assumption that non-HPITs mainly reflect the trades from uninformed liquidity-chasing traders, and HPITs are largely made by information-based traders. Similar observations are also found for MDAX stocks. However, for SDAX stocks, HPITs contribute less to daily price discovery than non-HPITs do. This could be due to the fact that the extremely wide bid-ask spread of SDAX stocks impedes price correction and incorporation of private information from informed trades. Having found that our identification method can effectively filter out informed trading, we then explore the practicability of our identification method by conducting a simple high frequency identification exercise for all stocks in our sample. We verify that the time required to identify informed trades based on our identification method is, on average, less than two milliseconds. Given that the average trade duration for most liquid DAX stocks is around 9 seconds, our identification approach also exhibits enhanced operating efficiency.

To further explore the market implications of HPITs, we investigate the relationship between HPITs and short-term volatility. Specifically, if HPITs mainly originate from informed traders, we should observe a negative relationship between HPITs and volatility, as predicted by the models of Hellwig (1980) and Wang (1993). Our results show that a stronger presence of HPITs does lead to a decline in volatility for most stocks in our sample, which strongly confirms the existence of information content in HPITs and their role as price stabilizers. In addition, we find that this negative effect on volatility increases with stocks' information transparency. ${ }^{13}$

We next look into the rationale behind this difference in volatility decline for the stocks in the three indexes. Specifically, we decompose HPITs into contrarian and herding (driving) HPITs, ${ }^{14}$

\footnotetext{
${ }^{13}$ We follow the literature and use the terms "information transparency" and "public disclosure" interchangeably.

${ }^{14}$ Contrarian HPITs refer to HPITs that trade against the current price trend. In a similar way, herding or driving HPITs are HPITs that follow or drive the actual price trend, respectively. In this study, we use the terms "driving" and "herding" interchangeably. The recent study by Van Kervel and Menkveld (2019) defines herding trades as with-wind trades.
} 
and conjecture that contrarian HPITs are more likely to be belief-based trades whereas herding HPITs are more likely to be fundamental-based trades. ${ }^{15}$ To date, the empirical literature has mainly focused on the behavior and implications of fundamental-based informed traders and mostly ignored the role and involvement of belief-based informed traders. With the increasing prevalence of uninformed trading, especially in the stocks with high public information disclosure and high liquidity, information about traders' motivations could also have significant implications on volatility. The model of Campbell et al. (1993) shows that a change in stock prices could occur due to information or liquidity-driven pressure. When the price change is due to fundamental information, price reversals are unlikely. However, price reversals are likely when price changes are caused by liquidity-driven pressure. This is precisely what we find with contrarian and herding HPITs. First, contrarian HPITs have a negative effect on return autocorrelation for most DAX, MDAX, and SDAX stocks. Second, herding HPITs have little effect on return autocorrelation for DAX and SDAX stocks and a positive impact on return autocorrelation for most MDAX stocks. In addition, our results show that the negative effect of contrarian HPITs increases with stocks' information transparency (i.e., it is smallest for SDAX stocks and highest for DAX stocks), which seems to confirm the dominance of uninformed liquidity trading and belief-based informed trading in DAX stocks, and an incentive for learning more about the trading motivations of other traders, in line with the prediction of Banerjee et al. (2018). ${ }^{16}$

Finally, we evaluate the impact of HPITs on short-term price efficiency for stocks traded in different information environments. We follow the recent empirical literature on price efficiency (see, for example, Boehmer and Kelley (2009), Chaboud et al. (2014), Conrad et al. (2015), and Rosch et al. (2016)), and use the variance ratio (Lo and MacKinlay (1989)) and the absolute value of autocorrelation as efficiency measures, which capture different aspects of price efficiency explored in the theoretical literature (Diamond (1985) and Gao and Liang (2013)). Specifically, short-term variance and autocorrelation capture the dynamic dimension of price efficiency by examining how closely the price follows a random walk and how predictable the returns are.

\footnotetext{
${ }^{15}$ Contrarian HPITs should, on average, reflect belief-based trades, and do not exclude fundamental-based trades. Accordingly, consider that fundamental-based traders are informed of good news and there is a simultaneous price decrease caused by selling pressure from uninformed liquidity traders. In this situation, both fundamental- and belief-based traders will trade against the current price decrease.

${ }^{16}$ Their theoretical model concludes that in a transparent market, greater public disclosure can discourage private learning about fundamentals while encouraging information acquisition about the trading motivation of others.
} 
After controlling for various market conditions, such as liquidity and volatility, we show that the presence of HPITs makes prices more efficient for DAX stocks, has an insignificant effect on price efficiency for MDAX stocks, and makes prices slightly less efficient for SDAX stocks. Our explanation is that in a market setting where both information transparency ${ }^{17}$ and liquidity are already high (DAX stocks), HPITs, which mainly reflect belief-based information, frequently react to uninformed trades and correct price distortion. As a result, price efficiency improves rapidly. In contrast, for stocks with less public disclosure and higher private informed trading (SDAX stocks), fundamental-based HPITs could lead to price inefficiency (e.g., price overreaction) without being corrected by belief-based HPITs because of a high trading cost. In addition, given that SDAX stocks feature a much wider bid-ask spread, the use of midquote price, as a proxy for expected true price, in price efficiency computation is also questionable.

The rest of the paper is organized as follows. Section 2 presents a literature review and our empirical hypotheses. Section 3 describes our dataset and the Xetra trading system. Section 4 investigates the existence of trade size clustering and the importance of LOB-matched trades. Section 5 examines the importance of roundedness and matchedness in informed trading identification. Section 6 assesses the impact of HPITs on volatility and identifies the underlying channels. Section 7 investigates the impact of HPITs on short-term price efficiency, and Section 8 concludes the paper.

\section{Literature Review and Main Hypotheses}

\subsection{Informed Trading and Matchedness}

In the theoretical models of Kyle (1985) and Admati and Pfleiderer (1988), informed, risk-neutral speculators endogenously take their price impact into account and trade strategically by spreading their trades over time and selecting the moments when market liquidity is high. Empirically, Barclay and Warner (1993) explore informed traders' choice of trade size, and are the first to

\footnotetext{
${ }^{17}$ Our paper distinguishes information transparency, which makes information acquisition about the fundamental value cheaper, from market transparency, which refers to how much information about the trading process is available to traders.
} 
propose and validate the well-known stealth trading hypothesis that informed traders concentrate their trades on medium sizes to conceal their information. They find that the cumulative stock-price change is due to medium-size trades. A generalized version of this hypothesis is that if informed traders are the main cause of convergence of the market price to the expected fundamental value, and if these traders concentrate their trades in certain specific types to hide their trading intentions, then most of a stock's cumulative price change should fall within these trade types. Consistent with Barclay et al. (1993), Chakravarty (2001) evaluates the stealth-trading hypothesis by further categorizing trade sizes by initiator (i.e., retail or institutional investors) and posits that institutions are informed traders. Several studies examine the link between stealth trading and trade clustering. ${ }^{18}$ Alexander and Peterson (2007) analyze trade size clustering with data from the NYSE and the NASDAQ, and suggest that rounded medium trade sizes have a greater price impact than do unrounded trades. Hodrick and Moulton (2005)study trade size clustering in a rational expectations framework and argue that when many heterogeneous uninformed investors are present, an asset will be traded at an increasing number of distinct sizes as investors' desire to trade exact quantities increases. Similarly, Moulton (2005) uses the data from foreign exchange markets to test the hypothesis that there is less trade size clustering shortly before the end of calendar quarters because portfolio managers seek to align their portfolios more fully with their given objectives. Moreover, the study provides evidence that the price impact of order flow is greater when customers care more about trading precise quantities. Garvey and Wu (2014) examine quantity choice patterns across trading hours and show that traders submit more non-rounded order sizes and more order sizes overall leading up to a day's market close. Studies that use roundedness to classify trades include those of Cai et al. (2006), Menkhoff and Schmeling (2010) and Ascioglu et al. (2011). The increasing number of distinct trade sizes might be related to another important dimension: matchedness, which measures responsiveness of liquidity demand of informed or uninformed traders with respect to

\footnotetext{
${ }^{18}$ Ball, Torous, and Tschoegl (1985) and Harris (1991) argue that while a more precise price that is mutually acceptable to both the buyer and the seller can be reached by continuing negotiations, the incremental benefit to each side decreases and the exposure of each side to reporting and price risk increases. As a result, clustering will occur as traders will seek to simplify the negotiation process. Another explanation is from a behavioral perspective. Wyckoff (1963) notes that traders think in round numbers and try to trade in round numbers. Niederhoffer and Osborne (1966) argue that the tendency of traders to prefer integers seems to be a fundamental and stable principle of stock market psychology. Ikenberry and Weston (2003) argue that price clustering may be a collective preference by investors to voluntarily trade at particular price levels in order to minimize cognitive processing costs.
} 
liquidity supply. In addition, given that the operations in financial market have become faster than ever before, matchedness provides insight into how high frequency traders are sensitive to liquidity. We conjecture that in a high public disclosure environment, as liquidity is already high, uninformed traders are likely to place more unmatched trades to meet their given objectives, and informed traders, who are sensitive to both liquidity and price, are likely to submit LOB-matched trades when correcting mispricing. However, in a low public disclosure environment, accompanied by a liquidity shortage, uninformed traders care more about liquidity and are likely to submit matched trades, and informed traders are likely to submit unmatched trades (e.g., marketable trades). In accordance with the existent literature and our conjecture, we are the first to consider matchedness as a trade attribute, and test the following hypothesis:

\begin{abstract}
H1: The information quality of matched trades, measured by the ratio of cumulative price contribution of matched trades to their corresponding proportion of volume, should be higher for stocks with greater public disclosure.
\end{abstract}

In this hypothesis high public disclosure stocks corresponds to the stocks with a large number of analysts, lower forecast dispersion among analysts and smaller forecast error.

\title{
2.2 Effect of Informed Trades on Price Volatility
}

Friedman (1953) argues that irrational investors destabilize prices by buying when prices are high and selling when prices are low, whereas rational speculators, by trading against irrational investors (buy when prices are low and sell when high), correct the deviation of prices from fundamentals and stabilize asset prices. Similarly, the noisy rational expectation models of Hellwig (1980) and Wang (1993) argue that volatility increases with uninformed or liquidity trading. Empirically, Avramov et al. (2006) document that the activities of both imitative and nonimitative investors have a significant effect on day-to-day volatility, although in different directions. At the intraday level, Blasco and Corredor (2017) examine the PIN measure and 
detect that informed trading is a price-stabilizing factor in heavily traded and highly capitalized stocks. Indirectly, using a monthly firm-level PIN measure and excess return, Lai et al. (2014) find a positive correlation between PIN and volatility in international markets. Therefore, we posit that:

\begin{abstract}
H2: If HPITs are related to informed trades, they should lead to a decline in volatility. Their negative effect on volatility should be greater for stocks with greater public disclosure.
\end{abstract}

\title{
2.3 Implications of Contrarian and Herding HPITs
}

Previous theoretical models follow Hayek's (1945) idea that prices aggregate fundamental-based information dispersed among market participants. In such markets, informed traders behave strategically and lead the market, as Kyle (1985) argues, and noise traders arrive in the market in an exogenous way. The introduction of noise trading in these models mainly aims to provide liquidity to informed traders, solving the "no trade" problem (Milgrom and Stokey (1982)), and imitating the real financial market. However, in practice, the proportion of uninformed traders depends on market information conditions and liquidity levels (Han et al. (2016)). Uninformed trading could also originate from financial institutions, ${ }^{19}$ and cause a significant impact on shortterm price formation (Cespa and Vives (2015)). Given the importance of uninformed trading in the financial markets, Han et al. (2016) propose an extended rational expectations equilibrium model in which the size of noise trading is endogenously determined by the population size of active liquidity traders in the market. They show that disclosing payoff-relevant public information weakens the information asymmetry problem, and lowers the expected loss of liquidity traders, thereby attracting more such traders. Under these circumstances, information about the trading motivations of other traders (i.e., belief-based information) become valuable. Given the presence of both informed and uninformed (liquidity) traders, Campbell et al. (1993) argue that, in a

\footnotetext{
${ }^{19}$ For example, Coval and Stafford (2007) find that in response to important redemption requests by clients, fund managers will curtail their positions and engage in "fire sales" for non-informational reasons. Additionally, Henderson et al. (2014) provide evidence that uninformed investment banks may also take large positions in commodity futures to hedge issuance of commodity-linked notes. Recently, with algorithmic trading data, Skjeltorp et al. (2016) find that algorithmic trading by large institutional investors is likely to be uninformed.
} 
quote-driven market, price reversals occur as risk-averse market makers absorb order flow from uninformed or liquidity traders. According to their model, a variation in stock price could be caused by informed or uninformed selling pressure. When price change is driven by information, price reversals are unlikely. However, if price change is driven by uninformed liquidity traders, price reversals are likely and liquidity suppliers should be compensated for taking this risk. In an open limit order book market where everyone could be a liquidity supplier when they know that the incoming orders originate from uninformed traders, especially for high learning capacity computers. Recently, Korajczyk and Murphy (2018) and Van Kervel and Menkveld (2019) show that it takes some time for high frequency traders to detect the fundamental-based trades, but ultimately they are able to identify the most informed traders and follow their trades. This allows us to test our third hypothesis:

H3.1: If contrarian HPITs mainly reflect belief-based information, they should have a negative effect on return autocorrelation and this negative effect should be more pronounced for stocks with greater public disclosure.

H3.2: If herding HPITs are mainly related to fundamental-based information, they should have an insignificant effect on return autocorrelation.

\subsection{Informed Trades and Short-term Price Efficiency}

Theoretical models (Diamond (1985), Gao and Liang (2013), Colombo, Femminis, and Pavan (2014), Banerjee et al. (2018), and Dugast and Foucault (2018), among many others), deduce price efficiency as a static precision of the conditional expected price based on fundamental information. Further, a subset of these papers focuses on a "crowding out" effect: greater public disclosure about fundamentals can crowd out private information acquisition, which in turn can reduce price informational efficiency. However, short-term price dynamics and price predictability are largely ignored. We extend the recent empirical literature (Boehmer and Kelley (2009), Chaboud et al. (2014), and Rosch et al. (2016)) on price efficiency to test the effect of HPITs on short-term price efficiency: 
H4: HPITs increase price efficiency for stocks with greater public disclosure and high levels of liquidity.

\section{Xetra Trading System, Ultra High-Frequency and Infor- mation Environment Data}

The data used in this study are from the Xetra trading system, which is operated by Deutsche Börse at the Frankfurt Stock Exchange (FSE); it has a similar structure to the Integrated Single Book of NASDAQ and Super Dot of NYSE. The Xetra trading system realizes more than $95 \%$ of the total transactions at German exchanges.

For highly liquid stocks, there is one dedicated market maker per stock and several sponsors during continuous trading. Similar to other stock trading systems, the Xetra trading system imposes a Price-Visibility-Time Priority condition, where the electronic trading system places the incoming order after checking the price and timestamps of all available limit orders in the LOB. Our database includes 20 levels of LOB information, ${ }^{20}$ which means that, by monitoring the LOB, any registered member can evaluate the liquidity supply dynamics and potential price impact of a market order. However, there is no information on the identities of market participants.

The reconstruction of the LOB is predominantly based on two main types of data streams: delta and snapshot. The delta tracks all the possible updates in the LOB such as entry, revision, cancellation, and expiration, whereas the snapshot gives an overview of the state of the LOB and is sent after a constant time interval for a given stock. Xetra original data with delta and snapshot messages are first processed using the XetraParser algorithm, developed by Bilodeau (2013). XetraParser reconstructs the real-time order book sequence including all the information for both auctions and continuous trading by implementing the Xetra trading protocol and Enhanced Broadcast. Then the raw LOB information is put in order and in a readable format for each update time. Useful and accurate information about the state of the LOB and the precise timestamp of order modifications and transactions during continuous trading are also retrieved.

\footnotetext{
${ }^{20}$ Fully hidden orders and the hidden part of an iceberg order are not observable in our dataset. However, as we observe the state of the LOB before and after the transaction, we can evaluate if a market order hits hidden orders or not. Our backtest results show that fewer than $3 \%$ of the market orders run into hidden orders, which represents about $6 \%$ of trade volumes.
} 
Our study focuses on the component stocks in three market indexes, DAX, MDAX, and SDAX, respectively. The DAX consists of the 30 major German companies listed on the Frankfurt Stock Exchange and is a blue-chip stock market index. MDAX includes 50 component stocks and is a stock index for the listed companies that rank below the companies in the DAX index in terms of market capitalization and order book volume (technology companies excluded). Finally, the SDAX is composed of 50 listed stocks that rank directly below the stocks in MDAX in terms of market capitalization and order book volume. There is a quarterly review to re-rank stocks among these three groups. Using data from the Compustat Global Security Daily files, Table 1 reports the descriptive statistics of daily market variables for DAX, MDAX, and SDAX stocks for six months, from February 1, 2013 to July 31, 2013. A decreasing monotonic trend is observed, from DAX to SDAX stocks, for all variables.

[Insert Table 1 here]

The high frequency LOB and trade data used in this study are registered with a timestamp in microseconds. Such precision allows us to identify the state of the LOB just before the trades and to determine whether the trade sizes are perfectly matched to the quantity standing in the open LOB. Table 2 presents the descriptive statistics of trades and information environment variables for DAX, MDAX, and SDAX stocks. To measure the information environment, we use the number of monthly news mentions and three analyst measures as the proxies of the information environment. The monthly number of news is the number of times that the company is mentioned in the news and social media registered by RavenPack. The analyst data are extracted from Institutional Brokers' Estimate System (I/B/E/S) for the period of 2011 to $2015 .^{21}$ We take the annual earnings per share (EPS) announcement as our target event. Following Barron et al. (1998), we first compute the number of analysts making forecasts about annual EPS up to the firm's actual announcement date. Second, we focus on the earning forecast dispersion, measured by the standard deviation of the forecasted EPS, standardized by the share price at the beginning of the year (Barron and Stuerke (1998) and Johnson (2004)). The third measure is the

\footnotetext{
${ }^{21}$ To obtain precise and reliable comparison results of different information environments, we select a more extended sample period of analyst data than that of our high frequency transactions and LOB data.
} 
forecast error, defined as the absolute difference between the mean forecast EPS and actual EPS, standardized by the price at the beginning of the year (Rajan and Servaes (1997) and Gu and Wang (2005)). ${ }^{22}$ We compute these three measures for all stocks and present the mean, median and standard deviation of three groups in Table 2. For a group with more transparent information environment, we expect to observe a larger number of analysts, lower forcast dispersion between analysts and smaller forecast error. It follows from Table 2 that DAX stocks are traded in a high public disclosure environment with low trading costs and high market transparency, while MDAX stocks are traded in a lower disclosure environment with higher trading costs and lower market transparency. Lastly, SDAX stocks feature the lowest disclosure environment and the highest trading costs.

[Insert Table 2 Here]

\section{Cross-sectional Analysis of Trade Size Clustering and LOB- Matched Volume}

We first investigate the existence of rounded size clustering in DAX, MDAX and SDAX stocks, and the importance of LOB-matched trades.

\subsection{Trade Size Distribution and Presence of Clustering}

We start by calculating trade size probability distributions for each stock in the three indexes, and then compute the average probability for each trade size across stocks in the same group. As mentioned above, the round-lot in the Xetra system is one unit; therefore, any integer number may appear in our sample. Figure 1 displays the histogram of trade sizes for DAX stocks (MDAX and SDAX stocks exhibit similar patterns). Three main insights arise from the figure: First, we

\footnotetext{
${ }^{22}$ For forecast dispersion and forecast error, we use the last forecast variables before the actual announcement.
} 
observe a decreasing trend, which suggests that the larger the trade size, the more infrequently it occurs. Second, the histogram features clustering on rounded numbers. For instance, there are more trades at 20 shares than 19 or 21 shares. Third, the clustering takes place in three levels. In increasing intensity, these levels are multiples of 10, 50 and 100. Specifically, when a trade size is a multiple of both 10 and 50, it will occur more frequently than a trade size that is a multiple of 10 only. For example, the trades at 150 shares occur more often than trades at 140 shares. The same logic applies to trade sizes that are multiples of 10,50 and $100 .^{23}$

\section{[Insert Figure 1 here]}

To test if our observations are statistically significant, we follow Alexander and Peterson (2007) and estimate the following regression separately for the three stock groups:

$$
\text { LnFreq }_{i}=\alpha+\beta_{5} D 5_{i}+\beta_{10} D 10_{i}+\beta_{50} D 50_{i}+\beta_{100} D 100_{i}+\beta_{\text {LnSize }} \text { LnSize }_{i}+\epsilon_{i},
$$

where $\mathrm{LnFreq}_{i}$ is the natural log of the percentage of trades of size $i, D 5_{i}, D 10_{i}, D 50_{i}, D 100_{i}$ are dummy variables if trade size $i$ is a multiple of 5, 10, 50,100, respectively, and LnSize $_{i}$ is the natural $\log$ of trade size $i$ measured in numbers of shares.

Panel A, B, and C of Table 3 reports the regression results of trade size clustering for DAX, MDAX, and SDAX stocks, respectively. The coefficients of dummy variables of 5, 10, 50 and 100 are all significant at the $5 \%$ level except the dummy variable of size 5 for DAX stocks. The coefficient of LnSize, which is significantly negative, confirms the negative slope of the histogram in Figure 1. In addition, a high value of adjusted $R^{2}$ suggests that the trade size pattern can be largely explained by trade size clustering and negative slope.

[Insert Table 3 here]

Table 4 presents the cross-sectional mean and standard deviation of clustered size across stocks and days. For each day, on average, the clustered trade-size percentage is significant and stable

\footnotetext{
${ }^{23}$ Our analysis does not rule out the possibility of the multiples of 10, 50 and 100 from high-frequency trading. We assume that traders choose roundedness when submitting their orders.
} 
with a mean of $33.39 \%$ and standard deviation of $3.97 \%$ for DAX stocks. Similar to the daily cross-sectional mean and standard deviation, the clustered rounded trade percentage remains stable across stocks, with a mean of $33.40 \%$, and standard deviation of $2.84 \%$. The same patterns are found for MDAX and SDAX stocks. The intuition is that traders' choices in terms of roundedness are consistent and remain stable among different groups.

\author{
[Insert Table 4 here]
}

\title{
4.2 LOB-matched Trades
}

In this subsection, we turn to another important dimension of trades, the matchedness of trade size to the quantities available in the LOB. A transaction is initiated by either the buy side or sell side. However, the counterparts of transactions are the limit orders standing in the open LOB.$^{24}$ With the development of information technology, the speed of submitting an order has become faster than ever before. For instance, Xetra implemented co-location service that allows traders to connect to the central server with much less latency (13 microseconds). Thus, with this speed advantage, traders can match the exact quantities standing in the open LOB when submitting a market or marketable orders. Note that a rounded trade size might be matched or unmatched depending on the quantity available in the open LOB. The dimension of matchedness is important because it provides insight into high frequency traders and their sensibility to liquidity. When traders have a speed advantage, they can time their trades and match the exact quantities available in the open LOB without making price impacts. According to Kyle (1985), informed traders camouflage themselves by splitting large volumes into small ones. Compared with the traders who passively split their desired trade volume, well-equipped high-frequency traders can now actively choose their submission time and trade the most favorable quantity available for them (quantity available at ask or bid side of the LOB). For our LOB-matchedtrade identification, one might argue that the marketable orders can also give the illusion of an

\footnotetext{
${ }^{24}$ This is not the case for the hidden orders which represent less than $5 \%$ of total trades in our sample, suggesting that our results are still robust.
} 
LOB-matched market order. As we show in Figure 2, a marketable bid (ask) order will both match the exact quantity in the ask (bid) side of the open LOB and increase (decrease) the best bid (ask) price to the price of the matched level. However, a simple buy (sell) market order will only consume the quantity standing in the ask (bid) side without creating a new best bid (ask) price. Therefore, to rule out the marketable orders, we also check the state of the LOB after the transaction to guarantee the accuracy of our matchedness identification.

[Insert Figure 2 here]

Table 5 reports the cross-sectional mean and standard deviation of matched trade sizes calculated at a daily level. For DAX stocks, the percentage of LOB-matched size is stable with a mean of $52.34 \%$, and the standard deviation of $5.76 \%$. At the stock level, the average percentage of LOB-matched size is $52.34 \%$ with a standard deviation of $4.59 \%$. For MDAX and SDAX stocks, we find similar cross-sectional and time-series patterns: the percentage of LOB-matched size exhibits a stable pattern for the groups with smaller trade sizes. It is interesting to see that more than half of the trade sizes seek to match the exact quantity available in the LOB to avoid price impact.

[Insert Table 5 here]

\subsection{Clustered and LOB-matched Trades}

Given the patterns and features of the rounded and LOB-matched trades, we consider these two dimensions together and investigate their dynamics across stocks. To do so, we consider the percentage of volumes of four trade types across stocks: UR-UM,UR-M, R-UM, R-M. ${ }^{25}$ As shown in Figure 3, the percentage of each trade size category is quite stable across stocks. Based

\footnotetext{
${ }^{25}$ The corresponding trade types are as follows: unrounded and unmatched trades (UR-UM), unrounded and matched trades (UR-M), rounded and unmatched trades (R-UM), and rounded and matched trades (R-M).
} 
on these observations, our interest now lies in the contribution of these trade types to price discovery, which is examined in next session.

[Insert Figure 3 here]

\section{Trade Types, HPITs, and Price Discovery}

\subsection{General Framework}

In order to address the issue of the contribution of different trade types to the price discovery process, ${ }^{26}$ we follow the rationale that if informed trades are the main cause of stock price changes and concentrate their trades in specific trade types, then most of a stock's cumulative price change should take place on these trade types. After the first empirical work of Barclay et al. (1993), an extensive body of literature attempts to identify informed trading with various datasets from different markets. For exemple, the studies categorize trade type by whether the trades are initiated by institutions (Chakravarty (2001)) or whether the trades feature oddlot sizes (O'Hara et al. (2014)). With our comprehensive dataset on the transaction and the open LOB, we can further evaluate how important the roundedness, and/or matchedness of trade is in informed trading identification. Also, we take trade size as our first dimension when distinguishing the trades. Trade-size is expressed in relative terms and defined as small, medium and large. The critical values used to categorize the different groups are the 30th and 70th percentiles of trade sizes.

We begin by examining the contribution of the rounded and matched trades separately, and then we consider these two dimensions together to get a clear insight into the price discovery contribution of each trade type. As in the study by O'Hara et al. (2014), we suppose there are $N$ trades for stock $s$ for day $t$, and each trade can be categorized into one of $J$ groups. In addition, we define the contribution of a given trade as the log difference between the trade's price and the

\footnotetext{
${ }^{26}$ We consider the daily price change as the total daily price discovery and take the ratio of the cumulated price change associated with a given category over the full price discovery as the contribution of that trade category.
} 
price of the previous transaction. ${ }^{27}$ The cumulative price contribution of the trades belonging to category $j$ for stock $s$ on day $t$ is defined as

$$
P C_{j}^{s, t}=\frac{\sum_{n=1}^{N} \delta_{n, j} r_{n}^{s, t}}{\sum_{n=1}^{N} r_{n}^{s, t}}
$$

where $\delta_{n, j}$ is an indicator variable that takes the value of one if the $n$th trade falls into size category $j$, and zero otherwise. Following Barclay and Warner (1993), we weigh each stock's price contribution to mitigate the problem of heteroskedasticity, which may be severe for firms with small cumulative changes. Suppose there are $N$ trades for stock $s$ on day $t$. The weight for stock $s$ on day $t$ is defined as

$$
w^{s, t}=\frac{\left|\sum_{n=1}^{N} r_{n}^{s, t}\right|}{\sum_{s=1}^{S}\left|\sum_{n=1}^{N} r_{n}^{s, t}\right|} .
$$

The weighted price contribution of trades in size category $j$ on day $t$ is defined as

$$
W P C_{j}^{t}=\sum_{s=1}^{S} w^{s, t} P C_{j}^{s, t}
$$

Suppose there are $T$ days in total. The weighted price contribution of trades in size category $j$ is defined as

$$
W P C_{j}=\frac{\sum_{t=1}^{T} W P C_{j}^{t}}{T} .
$$

To further quantify the contribution of different trade types to daily price discovery and identify HPITs, we estimate the following regression:

$$
P C_{j}^{s, t}=\sum_{j=1}^{k} \alpha_{j} \times \text { dummy }_{j}+\beta \times P c n t V o l u m e_{j}^{s, t}+\epsilon_{j}^{s, t}
$$

where $d u m m y_{j}$ is the dummy variable for category $j$ and PcntVolume ${ }_{j}^{s, t}$ relates to the volume

\footnotetext{
${ }^{27}$ Our method of computing the price contribution follows Barclay and Warner (1993) and Chakravarty (2001). By this definition, we also ascribe the contribution of the LOB between two consecutive trades to the second trade.
} 
percentage for stock $s$ on day $t$ in category $j$. The dependent variable is the cumulative price change of all trades in a given trade type divided by the cumulative price change of all trades during the period. The magnitudes of the denominator affect the level of this variable's sensitivity, especially for stocks with small cumulative price change. The weighting procedure can significantly reduce heteroskedasticity in the dependent variable.

\subsection{Trade Types and Price Contribution}

Using all transactions from all stocks, we first report the weighted price contribution associated with trade size as in Barclay and Warner (1993). Then we extend our analysis to the weighted price contribution of roundedness and matchedness ${ }^{28}$ separately. Finally, we provide a more detailed analysis by jointly considering trade size, roundedness and matchedness. To statistically identify HPITs, we estimate equation (6) with dummy variables of all 12 trade types (3 sizes $\times$ 2 roundedness $\times 2$ matchedness).

We take size as the first dimension for our trade classification and analysis of weighted price contribution. Specifically, for each stock, trades are first classified as small-, medium- or largesize according to the 30th and 70th percentiles of its own trade size distribution. Then, the corresponding proportion of trades and volumes, and price contribution are computed. Table 6 summarizes the results aggregated by size dimension for the DAX, MDAX and SDAX stocks. It follows that in our dataset it is the small-size trades that are associated with disproportionately large price changes relative to their proportion of volume. However, using a data sample between 1981 and 1984, Barclay and Warner (1993) find that medium-size trades are the trades associated with disproportionately large price changes relative to their proportion of volume. The difference between their findings and ours suggests a migration of informed trades from medium-size to small-size trades. One explanation is that trading cost decreases in financial markets. Informed traders always have to trade off between the gains related to their private information and the costs associated with the trading implementation. In previous quote-driven markets, traders paid, for each transaction, a high order processing cost charged by financial

\footnotetext{
${ }^{28}$ At this step of analysis, we keep size (e.g., small, medium, or large) as our first dimension.
} 
intermediaries. Thus, the practice of cutting large orders into small ones is very costly. However, the transformation from quote-driven to order-driven market and the introduction of electronic trading reduce dramatically this order processing cost and give informed traders an incentive to place more small orders. One may argue that the decrease in trading cost also gives incentive to liquidity traders to cut their orders. However, note that liquidity traders trade for exogenous discretionary reasons and are less sensible to trading cost decrease. In fact, they are ready to pay for liquidity. Therefore, we can safely link the liquidity cost decrease to the migration of informed trades from medium-size to small-size trades.

To extend our analysis of trade types to roundedness and matchedness, and further examine their corresponding marginal effects, we conduct a two-dimensional analysis by combining separately roundedness or matchedness with size (size-roundedness analysis vs. size-matchedness analysis). Table 7 shows the weighted price contributions of various trade types and the corresponding information quality for the DAX, MDAX and SDAX stocks. It is important to note that Table 7 presents the same findings as Table 6 does, but at a distributive level. To see this, consider that the small-size WPC for DAX in Table 6 (12.53\%) is the sum of the small-unrounded and small-rounded WPC for DAX in Panel A1 of Table $7(22.26 \%-9.73 \%=12.53 \%)$. In this typical example, we already notice that the small-rounded trade and small-unrounded trade do not have the same contribution to price discovery process. Therefore, considering all small-size trades in the same way without making any further distinction could be misleading. Panel A1 of Table 7 reports the price contribution of trade types based on size and roundedness for the DAX stocks, and suggests that the small-unrounded trade disproportionately contributes to the cumulative price change. ${ }^{29}$ Trades in the medium and large size, regardless of their roundedness, are less informative to the WPC. For instance, large-unrounded and large-rounded trades are responsible for $35.68 \%$ and $6.19 \%$ of the cumulative price change, respectively. However, these price changes also correspond to $20.03 \%$ and $9.71 \%$ of the total numbers of transactions, and $46.96 \%$ and $21.58 \%$ of the total trading volumes. Panels B1 and C1 report the price contribution of different trade types classified by size and roundedness for the MDAX and SDAX stocks. Similar to DAX stocks, small-unrounded trades contribute more to daily price discovery.

\footnotetext{
${ }^{29} \mathrm{By}$ taking the ratio of the WPC of a given category over the corresponding weight of such category in the total volumes and number of trades, we examine which category disproportionately contributes to the cumulative price change.
} 
Panels A2, B2, and C2 of Table 7 present the price contribution of different trade types according to size and matchedness. The results confirm our first hypothesis that in a high public disclosure environment, as liquidity is already high, uninformed traders are likely to place more unmatched trades to meet their given objectives, and informed traders, who are sensitive to both liquidity and price, are likely to submit LOB-matched trades when correcting mispricing. However, in a low public disclosure environment, accompanied by a liquidity shortage, uninformed traders care more about liquidity and are likely to submit matched trades, and informed traders are likely to submit unmatched trades (e.g., marketable trades). Specifically, for DAX stocks (Panel A2), the information quality of matched trades (1.17) is higher than that of unmatched ones $(0.82)$. The opposite is true for MDAX (0.66 for matched trades vs. 1.37 for unmatched trades) and SDAX (0.68 for matched trades vs. 1.23 for unmatched trades) stocks. However, for all three indexes, we observe a migration of informed trades from matched trades to unmatched ones when trade sizes increase. Take DAX stocks (Panel A2) as an example, with only about $2.68 \%$ of the total trade volumes, small-matched trades produce about $17.14 \%$ of the cumulative price change, which amounts to an information quality of 6.39. However, small unmatched trades, representing $2.73 \%$ of the total trade volumes, result in a WPC of $-4.61 \% .^{30}$ For medium-size trades, matched and unmatched trades have almost the same information quality. While for large-size trades, the information quality of matched trades is dominated by that of unmatched ones. Panels B2 and C2 report the price contribution of different trade types according to size and matchedness for MDAX and SDAX stocks. We observe slightly different but consistent results: 1) the information quality decreases when trade size increases. 2) Even for small-size group, information quality of matched trades does not dominate any more that of unmatched ones. Overall, our results suggest that matchedness, as a complement to roundedness, is an important dimension in informed trading identication.

In sum, the two-dimensional results of size-roundedness and size-matchedness provide evidence that both are important dimensions in informed trader identification. Further, informed trades are mainly associated with small-size trades, which is consistent with the stealth trading hypothesis.

\footnotetext{
${ }^{30}$ The negative WPC indicates that on average the associated trades run in the opposite direction to price.
} 
[Insert Table 7 here]

Now, we analyze WPC with all three dimensions: size, roundedness, and matchedness. Table 8 illustrates the WPC of the total of 12 trade types for DAX, MDAX and SDAX stocks. When we compare the ratio of the WPC to its corresponding weight in total trade volumes, the most informative trades for DAX stocks are small-unrounded-unmatched, small-unrounded-matched, smallrounded-matched, and medium-rounded-matched. Surprisingly, rounded-unmatched trades, regardless of their sizes, contribute negatively to cumulative price changes. Our conjecture is that for the DAX stocks, rounded-unmatched trades are predominantly initiated by liquiditychasing uninformed traders. The results show that the high level of granularity in our trade type analysis is important in informed trading identification. For example, without making distinction between rounded-matched and rounded-unmatched trades, Alexander and Peterson (2007) conclude that US stock markets feature size clustering and medium-rounded trades are more informative ones. For MDAX stocks, the results are similar, except we now find that the small-sized trades, regardless of trade roundedness and matchedness, contribute positively to daily price discovery, and, more importantly, medium-rounded-matched and medium-unroundedunmatched trades also contribute positively and disproportionately to price discovery. Again, for large-size trades, regardless of their roundedness and matchedness, there is no significant price contribution. For SDAX stocks, we have similar results as MDAX stocks, except that for medium-size trade, only medium-unrounded-unmatched trades make a signficiant contribution to price discovery. In summary, we show that several types of trade do contribute significantly to the daily price discovery process. This implies that informed traders choose different trade types to reveal their information according to the level of information disclosure.

[Insert Table 8 here] 


\subsection{HPIT Identification}

In previous section, we show that the inclusion of matchedness and roundedness in HPITs identification is important, and trade types of HPITs may also change across different indexes. We now turn to statistically identify HPITs for stocks in the three market indexes. To do so, we conduct the weighted-least-squares regressions of the percentage of the cumulative price change occurring in each trade type on dummy variables for the trade types and the percentage of volumes associated with that trade type (equation (6)). If there is no significant contribution of a given trade type to the daily price discovery process, the coefficient of the corresponding dummy variable should not be significantly different from zero. ${ }^{31}$

In Table 9, we present the regression results of the 12 trade types, classified by trade size, roundedness and matchedness, for the stocks in the three market indexes. A positive and significant coefficient for a dummy variable means that the price change related to such type moves in the same direction as a daily price change, while a negative and significant coefficient implies that the price change related to such type moves against the daily price change. For DAX stocks, we show that the coefficients of five dummy variables are significanlly different from zero at the $1 \%$ level. $^{32}$ The results imply that informed trades in DAX stocks concentrate in various smalland medium-size trade types. In addition, rounded-unmatched trades, regardless of their sizes, do not have contribution to daily price changes, which confirms what we observed in Table 8 . As for large-size trades, regardless of roundedness and matchedness, they do not contribute to the price discovery process. One explanation is that the decrease in the order processing cost encourages informed traders to split their trades into tiny pieces, and this is not the case for uninformed traders.

[Insert Table 9 here]

MDAX stocks have six positive and significant informed trade types which include the same five

\footnotetext{
${ }^{31}$ Note that our analysis focuses on the cumulative price changes of all trades in an associated trade type. Therefore, an estimated coefficient not significantly different from zero for a given type does not mean that there is no informed trades at all in such trade type. Instead, the associated trade type is dominated by uninformed trades.

${ }^{32}$ They are dummy variables for trade types of small-unrounded-unmatched, small-unrounded-matched, smallrounded-matched, medium-unrounded-unmatched, and medium-rounded-matched.
} 
informed trade types as DAX stocks and small-rounded-ummatched trades. As for SDAX stocks, there are only four positive and significant informed trade types. Specifically, for small-size trade types, the small-rounded-matched is the only one that is not informative to daily price, and for medium-size trade types, the medium-unrounded-unmatched is the only one that contributes significantly to the daily price changes. Again, large-size trades, regardless of roundedness and matchedness, do not belong to HPITs for any of the three groups. After identifying high price impact trades, we further attempt to investigate the informational quality of HPITs. The information quality is defined as the ratio of the WPC to its corresponding weight in the total trade volumes. ${ }^{33}$ Typically, we aggregate the trade types with positive and significant coefficients from the estimated results of equation (6) as aggregated HPITs. Then, we compute the corresponding aggregated WPC, the corresponding aggregated proportions in total trade numbers and total trade volumes, and take the number-based and volume-based information quality for the aggregated HPITs. Table 10 presents the number-based and volume-based information quality measures for DAX, MDAX and SDAX. One interesting insight is that both ratios decrease with market capitalization and public information disclosure, suggesting that HPITs for DAX stocks contribute more to the price discovery process. Specifically, on average, $1 \%$ of HPITs measured by volume contributes to $4.39 \%$ total price change for DAX stocks, $3.51 \%$ for MDAX and $3.00 \%$ for SDAX stocks.

Note that each HPIT type typically does not exclude uninformed trades: both informationrelated and uninformed trades could be present in any trade type. However, an HPIT category is the group in which the price contribution of information-related trades dominates that of uninformed traders. Therefore, these results on informational quality suggest that the dominance of information-related trades in such trade types is stronger for DAX stocks and weaker for SDAX stocks. In other words, market capitalisation, liquidity, and informational transparency are main factors to consider when informed traders choose trade types (size, roundedness, and matchedness) for their trades. For large-cap stocks exhibiting high liquidity and transparency, information-related traders are likely to submit small orders, whereas liquidity and uninformed traders are likely to use large-size orders because of a lower expected loss of liquidity (Han et

\footnotetext{
${ }^{33}$ We use number-based and volume-based information quality to refer to these two information quality measures.
} 
al.(2016)). However, regarding stocks with a liquidity shortage, information-related and uninformed traders have to consider the price impact of their trades, which limits their choice of trades in terms of size, roundedness, and matchedness. As a result, information-related and uninformed traders of less liquid stocks are likely to submit similar market orders, which are more likely to be found in the same trade types. ${ }^{34}$

[Insert Table 10 here]

\subsection{Intraday Dynamics of HPITs}

Once we have identified HPITs, we further evaluate their hourly contribution to price discovery during the trading day, as shown in Panel A of Figure 4. We first compute the hourly price contribution by taking the ratios of hourly price change over daily price change, and decompose the resulting hourly price contribution into those associated with HPITs and non-HPITs. For DAX stocks, the contribution to price discovery of HPITs dominates that of non-HPITs during the whole continuous trading session. More specifically, between 9 a.m. and 10 a.m. (during the beginning of a trading session), the contribution of HPITs is comparable to, but still higher than, that of non-HPITs (20\% vs. 15\%). Strikingly, non-HPITs produce about $15 \%$ of the daily price change with around $13 \%$ of total trading volumes. In contrast, HPITs contribute $20 \%$ of overall price change, with only $2 \%$ of total trading volumes. Also, for the time bins after 10 a.m., $82.64 \%$ of total price contributions come from HPITs. ${ }^{35}$ We examine another important factor in the identification of informed trading: the corresponding volumes for HPITs and non-HPITs. Panel B of Figure 4 shows the evolution of hourly trading volumes (expressed as a percentage of daily trade volumes) for HPITs and non-HPITs. The trading volumes of non-HPITs are much

\footnotetext{
${ }^{34}$ Note that based on our regression results, trade types of HPITs vary with stock indexes. In what follows, DAX HPITs contain small-unrounded-unmatched, small-unrounded-matched, small-rounded-matched, mediumunrounded-unmatched, and medium-rounded-matched trades. MDAX HPITs are composed of small-unroundedunmatched, small-unrounded-matched, small-rounded-unmatched, small-rounded-matched, medium-unroundedunmatched, and medium-rounded-matched trades. Finally, SDAX HPITs include small-unrounded-unmatched, small-unrounded-matched, small-rounded-unmatched, and medium-unrounded-unmatched trades.

${ }^{35}$ Table 11 reports that for the DAX index the total daily price contribution and the HPITs daily price contribution after 10 a.m are $53.14 \%$ and $64.30 \%$, respectively.
} 
larger than those of HPITs. More importantly, the hourly volumes of non-HPITs change a lot during the day and exhibit a strong seasonality pattern. That is, the highest trading volumes arrive at the beginning and the end of trading day. However, the hourly trading volumes of HPITs are around $2 \%$ per hour, which is relatively small, and quite stable during the day. The different intraday pattern of trading volumes for HPITs and non-HPITs suggests that uninformed liquidity traders are more likely to time their trades than are information-related traders.

[Insert Figure 4 here]

[Insert Table 11 here]

As Table 11 illustrates, a similar trend is found for MDAX stocks, but the dominance of HPITs over non-HPITs is less pronounced than that of DAX stocks. Surprisingly, for SDAX stocks, even though the information quality of HPITs is always higher than that of non-HPITs (2.997 vs. 0.693), the daily price contribution of HPITs is less than that of non-HPITs $(39.95 \%$ vs. $60.05 \%)$. One possible explanation is that information-related trades are impeded by a high trading cost, a serious obstacle faced by intraday traders. Generally, the net profit of intraday informed trades is the difference between the gains derived from their belief- or fundamentalbased information and the trading costs related to the order execution. In a market with a lower trading cost, information-related traders can get rewarded easily and have more incentive to trade against uninformed traders. In contrast, in a less liquid market that features a higher trading cost, informed traders have less incentive to trade against uninformed ones. To qualitatively investigate the relationship between trading cost and the contribution of HPITs across different markets, we present, in Figure 5, the intraday evolution of average relative bid-ask spread, which is defined as the ratio of bid-ask spread to midquote price. Two interesting insights arise from this figure. First, on average, the best bid-ask spread of SDAX stocks is much larger than those of DAX and MDAX stocks. More precisely, the spread of SDAX stocks is almost six times and three times as large as that of DAX and MDAX stocks, respectively. This means that informationrelated traders in SDAX stocks have to bear an extremely high cost before getting rewarded. Second, the average spreads for stocks in different indexes decrease during the trading day, with 
an exception in the middle of the trading session. These findings seem to confirm that: 1) most of the information is diffused at the beginning of the trading session; and 2) at the opening, the market exhibits a higher degree of information asymmetry, and liquidity providers face a high risk of adverse selection. To protect themselves, liquidity providers increase the bid-ask spread.

[Insert Figure 5 here]

\section{Impact of HPITs on Intraday Volatility}

\subsection{Impact on Intraday Volatility}

Up to now, we show how important HPITs are in daily price contribution and how to identify them. We next turn our attention to their implications for short-term volatilty. The noisy rational expectation model of Hellwig (1980) argues that volatility increases with uninformed trading. In his model, information is aggregated into price by the actions of risk-averse, heterogeneous agents who, individually, have no influence on prices. Rational informed investors stabilize prices by taking positions whenever prices deviate from their fundamentals, i.e. take long (short) position when the price is lower (higher) than fundamentals. As the proportion of informed investors increases, their impact on price increases, leading to a decrease in the deviation of price from its fundamental value. However, if the number of uninformed or liquidity traders increases, there will be an increase in volatility caused by uninformed trading. Wang (1993) also provides a model of asymmetric information and shows that the conditional volatility of prices increases with uninformed trading. In sum, both Hellwig's (1980) and Wang's (1993) models show that volatility decreases with informed trading and increases with liquidity/uninformed trading. Therefore, if HPITs are associated with informed trading, our results should be in line with the theoretical models. That is, we should find that an increase in HPITs leads to a decrease in volatility. In addition, because it is hardly possible for traders' risk aversion and market settings to change on a daily basis, it is possible to rule out such "macro factors" and reasonable to consider in terms of a close connection between information trading and volatility. 
In order to examine the impact of HPITs on intraday volatility, we analyze the effect of the proportion of HPITs on the 15-min conditional volatility. Given that high-frequency data behaves very differently from low-frequency data, before estimating the model, we first remove seasonality by following a regression approach as did Dufour and Engle (2000). Moreover, the Ljung-Box statistics with 15 lags on the deseasonalized returns and the corresponding volatilities reject independence at all significance levels for most of the stocks in the sample. Thus, taking the model efficiency and parsimony into consideration, we estimate the model with an $\operatorname{EGARCH}(1,1)$ for all DAX, MDAX, and SDAX stocks:

$$
\begin{gathered}
r_{i}=\sigma_{i} \cdot \varepsilon_{i} \\
\log \left(\sigma_{i}^{2}\right)=\omega+\sum_{j=1}^{p} \alpha_{j} g\left(Z_{i-j}\right)+\sum_{j=1}^{q} \beta_{j} \log \left(\sigma_{i-j}^{2}\right)+\gamma H P I T \%_{i-1}
\end{gathered}
$$

with $g\left(Z_{i}\right)=Z_{i}+\lambda\left(\left|Z_{i}\right|-E\left(\left|Z_{i}\right|\right)\right)$, and where $r_{i}$ is $i$ th 15-min deseasonalized return, $H P I T \%_{i-1}$ relates to the proportion of HPITs for the period $i-1$, and $\varepsilon_{i}$ is a normally distributed random variable. The parameters $\beta$ and $\lambda$ capture the autocorrelation in volatility. $\gamma$ measures the impact of HPITs on volatility. After the estimation, the model is validated again by Ljung-Box statistics (with 15 lags) of the standardized residuals and squared standardized residuals.

Table 12 shows the estimation results of the proposed model for DAX stocks. The results suggest that 1) there is a high persistence in volatility given that the parameter $\beta$ has a mean of 0.862 . 2) 29 out of 30 DAX stocks have a negative $\gamma$, statistically significant at the $1 \%$ level. 3) the proposed model effectively captures the dynamics of volatility, which is validated by Ljung-Box statistics. ${ }^{36}$ Similar results are obtained for the MDAX and SDAX stocks. For the sake of brevity, we only present a summary of the estimated parameters in Table 13, instead of full estimation results. In sum, HPITs have negative effect on volatility. However, this negative effect varies across different stock indexes. Specifically, this negative effect decreases, in the absolute term,

\footnotetext{
${ }^{36}$ The $5 \%$ critical value for the Ljung-Box with 15 lags is 24.99 . A statistic less than 24.99 means that we cannot reject the null hypothesis that the time series is autocorrelated.
} 
from 2.24 for DAX stocks to 1.11 for MDAX stocks, and 0.42 for SDAX stocks.

[Insert Table 12 here]

[Insert Table 13 here]

One plausible explanation for this difference in the impact on volatility is the difference in information condition of DAX, MDAX, and SDAX stocks. More specifically, DAX stocks are large-cap stocks and have greater public disclosure, which leads to favorable trading conditions such as more transparency, higher liquidity and more market turnover. The channel under which more disclosure leads to more market turnover and liquidity trading is highlighted in the theoretical model of Han et al. (2016). The intuition is that greater public disclosure lowers the expected loss of liquidity traders, thereby attracting more such traders. Implicitly, the proportion of traders that approximately know the (expected) fundamental value of the stocks is higher than that for the less transparent MDAX and SDAX stocks. Furthermore, a greater public disclosure market discourages private learning about fundamentals but encourages information acquisition about trading motivations of other traders (Banerjee et al. (2018)). Therefore, traders have a greater incentive to identify the trading motivation of other traders. For example, when the stock price deviates from its (expected) fundamental value caused by buying or selling pressure from uninformed or liquidity traders, belief-based traders will take the contrarian trades by executing sell or buy trades to correct this price deviation. Also, because of the favorable trade condition in the DAX stocks such as smaller bid-ask spread and a large number of uninformed traders, informed traders can easily get rewarded by providing liquidity to uninformed traders.

Generally, as mentioned above, belief-based traders do not rule out traditional or sophisticated high-frequency market makers who have access to both public information and information related to their customers. In the modern financial market, sophisticated market making algorithm can learn fast about trading motivations of other traders by preying on the footprints they leave in the market (Korajczyk and Murphy (2018) and Van Kervel and Menkveld (2019)). In addition, having all the trading history of their customers and participating actively in the interdealer 
markets, the market makers possess private information about other market participants and are in a good position to judge if someone is informed or not. ${ }^{37}$

However, for less liquid medium-cap stocks there is more information asymmetry and a larger bidask spread. Thus, the proportion of uninformed traders is lower compared with large-cap DAX stocks, and the short-term price deviations caused by liquidity trading are less likely. However, even if this price distortion still exists, it is difficult for informed traders to get rewarded when faced with a large transaction cost (a large bid-ask spread). The same arguments also hold for the least liquid SDAX stocks. That is, SDAX stocks are characterized by the highest bid-ask spread, the lowest proportion of uninformed traders, the least likely short-term price deviations. Overall, in line with previous theoretical models, the negative relationship between HPITs and intraday volatility suggests that HPITs are rewarded for acting as price stabilizers. However, this negative effect decreases when information asymmetry and bid-ask spread increase, which are consistent with the idea that informed traders have to limit their involvement as price stabilizer in presence of unfavorable trade conditions.

\subsection{Information Conveyed by HPITs: Autocorrelation Test}

Thus far, we have empirically shown that a higher proportion of HPITs leads to a decline in volatility. We now provide more detailed empirical evidence on the questions of which type of informed trading could reduce volatility and how the magnitude of this decline in volatility varies with information environments. To do so, we define contrarian (herding) HPITs as trades that are against (after) the current price trend. Specifically, buy (positive) HPITs in the presence of decreasing price and sell (negative) HPITs during a price increase are designated as contrarian HPITs. Similarly, buy (positive) HPITs during a price increase and sell (negative) HPITs in the presence of a price decrease are defined as herding HPITs. By definition, contrarian (herding) HPITs are related to belief-based (fundamental-based) informed trading. If our conjecture that HPITs are sent by informed traders (fundamental-based and belief-based) is correct, we should

\footnotetext{
${ }^{37}$ Trading ahead of one's own customers is illegal, but the mentioned strategy entails trading after or against one's customers.
} 
have negative coefficients for contrarian HPITs, and insignificant ones for herding HPITs in equation (9). That is, contrarian HPITs lead to a price reversal and herding HPITs have no effect on return autocorrelation. In addition, $\delta_{1, i}+\delta_{2, i}$ capture the net effect of informed trading behavior in a given information environment. More specifically, when $\delta_{1, i}+\delta_{2, i}<0$, this suggests that informed traders concentrate more on trading against uninformed traders than trading with their own private information about fundamentals. The opposite is true when $\delta_{1, i}+\delta_{2, i}>0$. We estimate the following regression by controlling for the trading volume ${ }^{38}$ and other market variables:

$$
\begin{aligned}
R_{i, t}= & \left(\delta_{1, i} H_{P I T} \%_{i, \mathrm{t}-1} * 1_{(\text {contrarian })}+\delta_{2, i} \text { HPIT }_{i, \mathrm{t}-1} * 1_{(\text {herding }}+\delta_{3, i} \text { Volume }_{t-1}\right) R_{i, t-1} \\
& +\psi_{i, 1} \text { Range }_{i, t-1}+\psi_{i, 2} \text { Spread }_{i, t-1}+\psi_{i, 3} \text { Price }_{i, t-1} \\
& +\sum_{k=2}^{5} \beta_{i, k} R_{i, t-k}+\sum_{k=1}^{N} \alpha_{i, k} D_{k t}+e_{i, t}
\end{aligned}
$$

where Range $_{i, t-1}$ is the range between maximum and minimum price for stock $i$ during the period $t-1$. Spread $_{i, t-1}$ and Volume $_{t-1}$ are the average spread and the total trade volume during the period $t-1$, respectively. $\delta_{1, i}$ and $\delta_{2, i}$ measure the impact of contrarian and herding HPITs on return's autocorrelation. Moreover, $1_{\text {(contrarian) }}$ and $1_{\text {(herding) }}$ are dummy variables that take the value of 1 if the price change of the HPITs goes against or follows the price trend at $t-1$. Finally, $D_{k t}$ is the dummy variable that takes the value of 1 if period $t$ belongs to hour $k$ of the trading session. Table 14 presents the results for DAX stocks. The estimated $\delta_{1}$ for contrarian HPITs are negative and significant for all stocks at the $1 \%$ level of confidence. Thus, contrarian HPITs of the DAX stocks do lead to price reversals. However, $\delta_{2}$ has an average around zero and 27 out of 30 stocks have insignificant coefficients, which suggests an insignificant effect of herding HPITs on return for the DAX stocks. The total effect of HPITs, measured by $\delta_{1}+\delta_{2}$, is also negative and significant for 27 out of 30 stocks, which is consistent with the result that HPITs lead to a decline in volatility. Therefore, based on these empirical results, we conclude that in a high-level public information environment, HPITs mainly capture belief-based information rather than fundamental-based information.

\footnotetext{
${ }^{38}$ In particular, Volume is included in regression 9 to control for result of Campbell et al. (1993) that volume contributes to return reversals.
} 
[Insert Table 14 here]

For the sake of brevity, we present a summary of the estimated parameters for stocks in the three indexes in Table 15, instead of full estimation results. For MDAX stocks, both coefficients of contrarian and herding HPITs have an impact on short-term returns. Specifically, all stocks have negative and significant $\delta_{1}$, and 29 out of 50 stocks have positive and significant $\delta_{2}$. This result suggests that herding HPITs lead to a price continuation for more than half of MDAX stocks. ${ }^{39}$ In terms of total effect, 45 out of 50 stocks have negative and significant $\delta_{1}+\delta_{2}$, which confirms the dominance of fundamental-based information. From the perspective of information acquisition, the results also provide support for complementarity in learning: in a less public information disclosure environment when prices do not fully reflect available public information, traders have the incentive to learn both dimensions of information and make profits by incorporating fundamental-based information into the current price or correcting price distortion created by uninformed traders. Finally, for SDAX stocks, 31 out of 48 stocks have negative and significant $\delta_{1}$ for contrarian HPITs, which presents the lowest ratio of negative and significant $\delta_{1}$ among three indexes. Also, 40 out of 48 stocks have insignificant $\delta_{2}$ for herding HPITs, which suggests that herding HPITs have little effect on return autocorrelation.

[Insert Table 15 here]

Information-related trades can occur in two situations, one is when the market price deviates from its fundamental price, and actual spread allows informed traders to get rewarded by correcting the price distortion. The second one is when the informed traders have private information and try to take the maximum profit before this private information becomes public. For large-cap stocks such as DAX stocks, the first situation is more often observed. For SDAX stocks, the second situation is more likely. The MDAX stocks are in-between. Our results show that 1) the average effect of HPITs for DAX stocks is -0.903 , suggesting that a one-percent increase in HPITs would reduce the autocorrelation by approximately 0.01 on average; 2) The effect is less

\footnotetext{
${ }^{39}$ The theoretical model of Kyle (1985) does not imply the autocorrelation of return in the presence of informed traders. However, empirically, due to market friction or information quality, we could observe a positive autocorrelation.
} 
pronounced for MDAX stocks with decreases of 0.0036 , and the smallest (0.0013) for the SDAX stocks. In sum, our results of autocorrelation test provide more evidence that contrarian HPITs act as a price stabilizer and lead to return reversal for DAX, MDAX and SDAX stocks. However, the size of this reversal decreases when information asymmetry and bid-ask spread increase.

\section{Impact of HPIT on Price Efficiency}

So far, we have empirically shown that HPITs lead to a decline in intraday volatility by making more contrarian trades, and explained why this decline in volatility is not the same across different groups identified by the difference in their information setting. We next turn our attention to the effect of HPITs on price efficiency. One of the fundamental roles of the financial market is to facilitate the price discovery process, which also means making stock prices to reflect fundamentals quickly. Thus the questions of to what the extent prices reflect the fundamental values and how price efficiency is affected by various market settings have drawn strong interest among academics, practitioners, and regulators. However, theoretical and empirical finance do not always have the same measurements and conclusions of market efficiency, depending on their focus. Specifically, theoretical models emphasize on the static precision of the conditional expected price based on fundamental information (Diamond (1985), Gao and Liang (2013), Colombo et al. (2014), Banerjee et al. (2018), and Dugast and Foucault (2018)), while empirical studies attempt to assess the dynamics aspect of efficiency, that is, statistically, how closely stock prices follow a random walk (Lo and MacKinlay (1988), Boehmer and Kelly (2009), Chaboud et al. (2014), Conrad et al. (2015), and Rosch et al. (2016)). Given this nuanced divergence in measurement, our study follows the empirical finance literature and uses variance ratio- and autocorrelation-based measurements for price efficiency. 


\subsection{Variance Ratio Evidence}

The first measurement we use for price efficiency is derived from the variance ratio proposed by Lo and MacKinlay (1989). According to their notation, $x_{t}$ represents a log price process, ${ }^{40}$ and there are $n$ non-overlapping long-horizon intervals in the measurement interval and $q$ non-overlapping short-horizon intervals in each long-horizon interval. Moreover, each interval is equally spaced so that there exist $T=n q$ returns in the measurement interval. In such a setting, the estimate of the mean drift in prices is equal to:

$$
\hat{\mu}=\frac{1}{n q} \sum_{k=1}^{n q}\left(x_{k}-x_{k-1}\right)=\frac{1}{n q}\left(x_{n q}-x_{0}\right),
$$

and the estimates of the variance are as follow

$$
\begin{gathered}
\bar{\sigma}_{a}^{2}(q)=\frac{1}{n q-1} \sum_{k=1}^{n q}\left(x_{k}-x_{k-1}-\hat{\mu}\right)^{2} \\
\bar{\sigma}_{c}^{2}(q)=\frac{1}{m} \sum_{k=q}^{n q}\left(x_{k}-x_{k-q}-q \hat{\mu}\right)^{2}
\end{gathered}
$$

where $m=q(n q-q+1) \times\left(1-\frac{q}{n q}\right)$, and $\bar{\sigma}_{a}^{2}$ and $\bar{\sigma}_{c}^{2}(q)$ are short and large interval return variances, respectively.

If prices follow a random walk process, variances should be linear in the measurement interval. This implies that the ratio of scaled large interval return variance over short interval return variance, $\bar{\sigma}_{c}^{2}(q) / \bar{\sigma}_{a}^{2}$, should be equal to one. Specifically, the test based on the random walk hypothesis is

$$
M_{r}(q) \equiv \frac{\bar{\sigma}_{c}^{2}(q)}{\bar{\sigma}_{a}^{2}}-1=0 .
$$

For our dataset, we take 30 seconds and 5 minutes as our short and large intervals, respectively. Implicitly, $q$ is equal to 10 . Further, we compute the ratio of variance over 2-hour and 4-hour

\footnotetext{
${ }^{40}$ We use midquote price instead of trade price to avoid the negative autocorrelation caused by the bid-ask bounce.
} 
measurement intervals. Precisely, taking the example of a 2-hour measurement interval, there are 240 non-overlapping short and 24 non-overlapping large intervals. To avoid the degeneration of the variance ratio, we require at least 30 nonzero short interval returns in each 2-hour measurement interval. We choose 30 seconds as our short intervals because the interval should be short enough to capture the high-frequency dynamics in price changes and provide sufficient observations to compute the variance. This interval also needs to be long enough to avoid high-frequency noise.

To examine the effect of HPITs on price efficiency, we take the absolute value of $M_{r}(q)-1$ as our efficiency measure and run the following fix-effect and random-effect panel regressions:

$$
\begin{aligned}
\left|M_{r}(q)-1\right|_{i, t}= & \alpha_{i}+\gamma_{t}+\beta_{1} \times \operatorname{HPIT}_{i, t-1}+\beta_{2} \times \log \left(\text { Price }_{i, t-1}\right) \\
& +\beta_{3} \times \text { Range }_{i, t-1}+\beta_{4} \times \operatorname{Spread}_{i, t-1}+\epsilon_{i, t} \\
\left|M_{r}(q)-1\right|_{i, t}=\mu & +\alpha_{i}+\gamma_{t}+\beta_{1} \times \operatorname{HPIT}_{i, t-1}+\beta_{2} \times \log \left(\text { Price }_{i, t-1}\right) \\
& +\beta_{3} \times \text { Range }_{i, t-1}+\beta_{4} \times \operatorname{Spread}_{i, t-1}+\epsilon_{i, t} .
\end{aligned}
$$

As mentioned above, we include Range to control for volatility and Spread for liquidity. If HPITs are information-related trades, according to the random walk hypothesis, the future price should be less predictable because more information is incorporated in the price. In other words, the presence of HPITs helps to incorporate information into the price and will make the future prices less predictable or more likely to follow a random walk process. Our dependent variable is the absolute value of $M_{r}(q)-1$ and the minimum value of zero corresponds to a pure random walk process. Therefore, if our conjecture is correct, we expect a negative effect of HPITs on the dependent variable.

Table 16 reports the results of regressions (14) and (15). For DAX stocks, an increase in HPITs significantly results in price efficiency at the $1 \%$ level. However, this effect decreases and becomes insignificant for MDAX stocks. Although the presence of more HPITs still makes the future price more efficient, it is not statistically significant at the $5 \%$ level. Finally, regarding the least liquid 
SDAX stocks, more HPITs adversely affect price efficiency and make it more predictable at the $5 \%$ level. Furthermore, as mentioned above, informed traders consider both expected profits and trading costs before submitting their orders. It follows from Table 16 that an increase in the spread also makes the future price more efficient for both DAX and MDAX stocks at the $1 \%$ level. However, for the least liquid SDAX stocks, which already have extremely wide bid-ask spread, the change in spread has little impact on efficiency.

[Insert Table 16 here]

We draw two main inferences from the results. First, both HPITs and bid-ask spread play a role in price efficiency. More specifically, an increase in HPITs makes the price more efficient by adding more information into the price and make it less predictable. However, another channel through which price efficiency can be improved is to increase the bid-ask spread. To understand the relationship between spread and price efficiency, consider that the expected fundamental value of the stock is $p_{0}$, which is different from the current midquote price $m q_{0}$, and there exists a spread $s_{0}$ between the best ask and the best bid price. When $s_{0}$ is so large that the expected fundamental price, $p_{0}$, falls in the interval $\left(m q_{0}-\frac{s_{0}}{2}, m q_{0}+\frac{s_{0}}{2}\right)$, this discourages informationrelated trades because the gain from the information cannot cover the transaction cost. As a result, prices remain efficient without trading activity.

The second inference we draw from these results is that the way information is incorporated in the price varies with liquidity levels and information environments. Combined with the results found in subsection 6.2 for DAX stocks, HPITs mainly reflect contrarian trades initiated by belief-based traders. Given greater public disclosure, competitiveness between belief-based traders is high. When prices deviate from the expected fundamental value, these informed-traders have to react quickly to get rewarded. Because this process makes more information be incorporated in the stock price, price efficiency is improved rapidly. However, for illiquid stocks, herding HPITs might be followed by uninformed trades, and a high bid-ask spread impedes the price correction from contrarian HPITs. In consequence, price efficiency deteriorates, and prices are less likely to follow a random walk. Another explanation for this adverse effect is that the expected fundamental 
prices may fall in the interval $\left(m q_{0}-\frac{s_{0}}{2}, m q_{0}+\frac{s_{0}}{2}\right)$, but the efficiency is only computed with the mid-quote price, which might not be a reliable proxy for the expected fundamental value when the bid-ask spread is wide.

\subsection{Autocorrelation Evidence}

The variance ratio measures only one facet of price informational efficiency. More generally, one concern about high-frequency information-related traders is that they cut their large volumes into small ones and span them during a longer horizon, which may cause autocorrelation. We thus access the impact of HPITs on a more general measure of price efficiency: the autocorrelation of high-frequency return. Specifically, we investigate the effect of HPITs on the absolute value of the first-order autocorrelation of five-second returns every two hours. If HPITs are informationrelated trades, the returns should be less autocorrelated because more information is incorporated in the price, which suggests a negative effect of HPIT on absolute autocorrelation coefficient. The following panel regression is estimated for all DAX, MDAX, and SDAX stocks:

$$
\begin{aligned}
|\rho|_{i, t}= & \alpha_{i}+\gamma_{t}+\beta_{1} \times \operatorname{HPIT}_{i, t-1}+\beta_{2} \times \log \left(\text { Price }_{i, t-1}\right) \\
& +\beta_{3} \times \text { Range }_{i, t-1}+\beta_{4} \times \text { Spread }_{i, t-1}+\epsilon_{i, t} \\
|\rho|_{i, t}=\mu & +\alpha_{i}+\gamma_{t}+\beta_{1} \times \text { HPIT }_{i, t-1}+\beta_{2} \times \log \left(\text { Price }_{i, t-1}\right) \\
& +\beta_{3} \times \text { Range }_{i, t-1}+\beta_{4} \times \text { Spread }_{i, t-1}+\epsilon_{i, t}
\end{aligned}
$$

Table 17 presents the results of a panel regression of the absolute autocorrelation coefficient on HPITs and other market variables. Similar to the results for the variance ratio, for DAX stocks, a higher proportion of HPITs decreases the intraday return autocorrelation. This effect is significant for both fixed effect and random effect panel regressions. A less significant effect is found for MDAX stocks and an insignificant effect for SDAX stocks. Specifically, HPITs of SDAX stocks have a positive impact on autocorrelation, which confirms the slight price deviation 
after fundamental-based HPITs.

The results on autocorrelation-based efficiency provide more evidence on how HPITs act as a price stabilizer for the DAX, MDAX, and SDAX. Specifically, this effect depends on the characteristics of trading and information environments. For large-cap, liquid stocks such as DAX stocks, beliefbased trades reduce the return autocorrelation, whereas for medium- and small-cap stocks, given that a wide bid-ask spread impedes price correction, the role played by HPITs as price stabilizer is less pronounced. As a result, lesser price efficiency is observed in MDAX and SDAX.

[Insert Table 17 here]

\section{Conclusion}

We identify trades that have disproportionately large cumulative price changes relative to their proportion of volume (HPITs), and relate them to informed trades. Our results suggest that HPITs might contain information about either the fundamental value of stocks (fundamentalbased) or the trading motivations of other traders (belief-based), or both, depending on stocks' information environments. We also show that the market implications of HPITs vary significantly with information environments. Typically, we consider stocks in the DAX, MDAX and SDAX indexes, traded at the Frankfurt Stock Exchange, as a natural setting in terms of information environments to study the type of information conveyed by HPITs.

To precisely identify HPITs, we first confirm the existence of trade size clustering and LOB matchedness. Typically, trade sizes feature clustering on 10, 50, 100 or their multiples, which accounts for more than $40 \%$ of total trades, and LOB-matched trades represent more than $50 \%$ of total trades. In addition, our empirical results of matched trades confirm our first hypothesis that the information quality of matched trades should be higher for stocks with greater public disclosure. The two-dimensional results of size-roundedness and size-matchedness provide evidence that both are important dimensions in informed trading identification. 
With the above identified informed trading, we validate the second hypothesis that a stronger presence of HPITs leads to a decline in volatility for all three groups of stocks. However, this negative effect increases with the level of stocks' public disclosure (i.e., it is highest for DAX stocks and lowest for SDAX stocks). To further explore the information conveyed by HPITs, we decompose HPITs into contrarian and herding HPITs, and show that contrarian HPITs are responsible for this decline in volatility and lead to price reversals. Again, this negative effect increases with the level of stocks' public disclosure. Our empirical findings support the third hypothesis that 1) contrarian HPITs are more present in the greater public disclosure market and trade against uninformed traders; and 2) herding HPITs are mainly related to fundamentalbased information and have an insignificant effect on return autocorrelation.

Finally, we use variance-ratio and autocorrelation-based price efficiency measures to test our fourth hypothesis that HPITs increase price efficiency for stocks with greater public disclosure and high levels of liquidity. Our results show that for DAX stocks, the increase in HPITs improves price efficiency significantly. Similar results are found for MDAX stocks, but are not significant. For the least liquid SDAX stocks, the presence of HPITs slightly reduces market efficiency. We provide two possible explanations for this phenomenon: First, for stocks with more privateinformation traders and a wide bid-ask spread, fundamental-based informed trades might be followed by uninformed trades that lead a price to deviate from its fundamental level. Further, a high bid-ask spread impedes the price correction made by belief-based traders. Therefore, price efficiency deteriorates. Second, the midquote price we use to compute the efficiency measure might not be a reliable proxy for the expected true price, especially for the least liquid stocks with a large bid-ask spread. More research is needed to find a better proxy. 


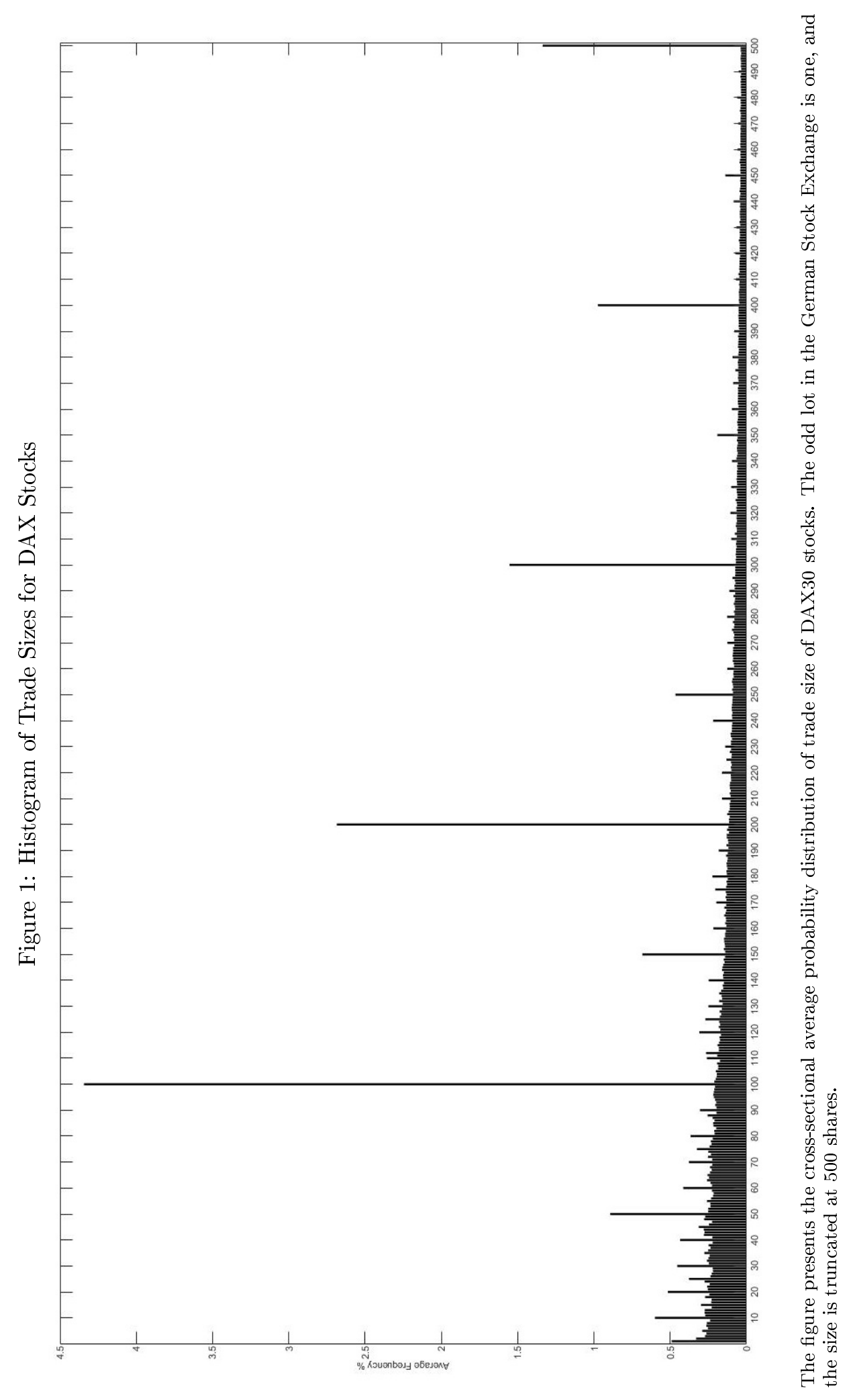


Figure 2: Marketable Orders Vs. Market Orders

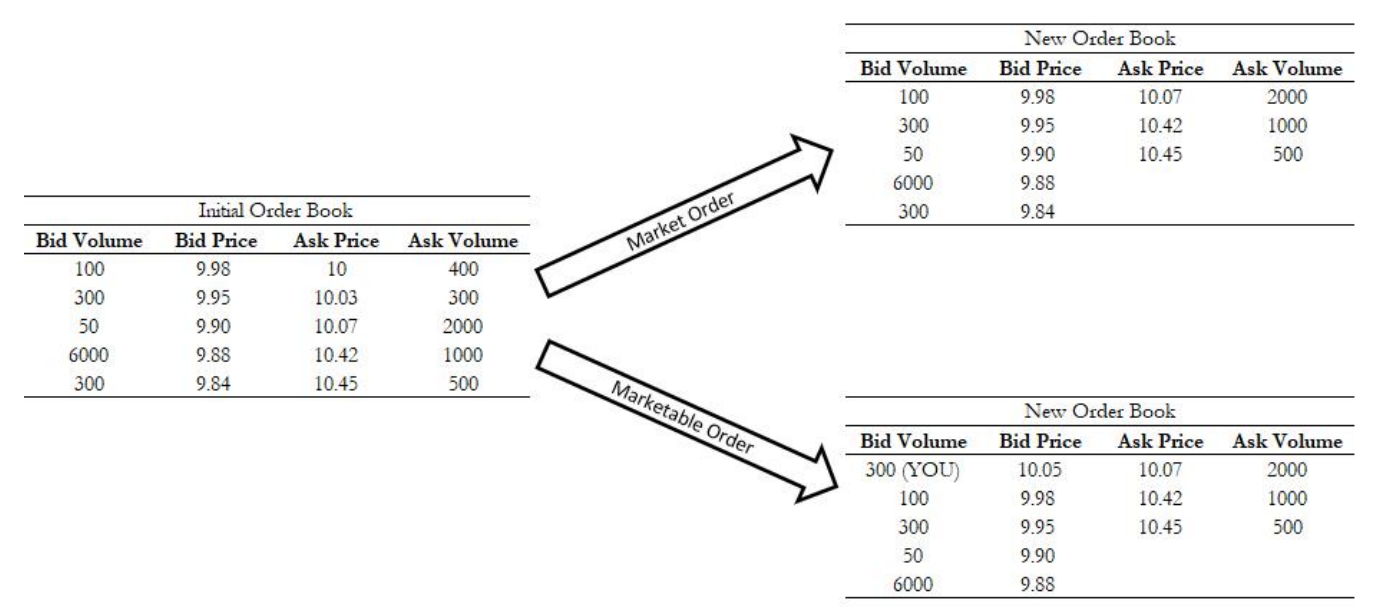

The figure presents and compares the different outcomes of market and marketable orders. The market order is submitted with volume of 700 shares. The marketable order is submitted with volume of 1000 shares and price of 10.05 Euros. 


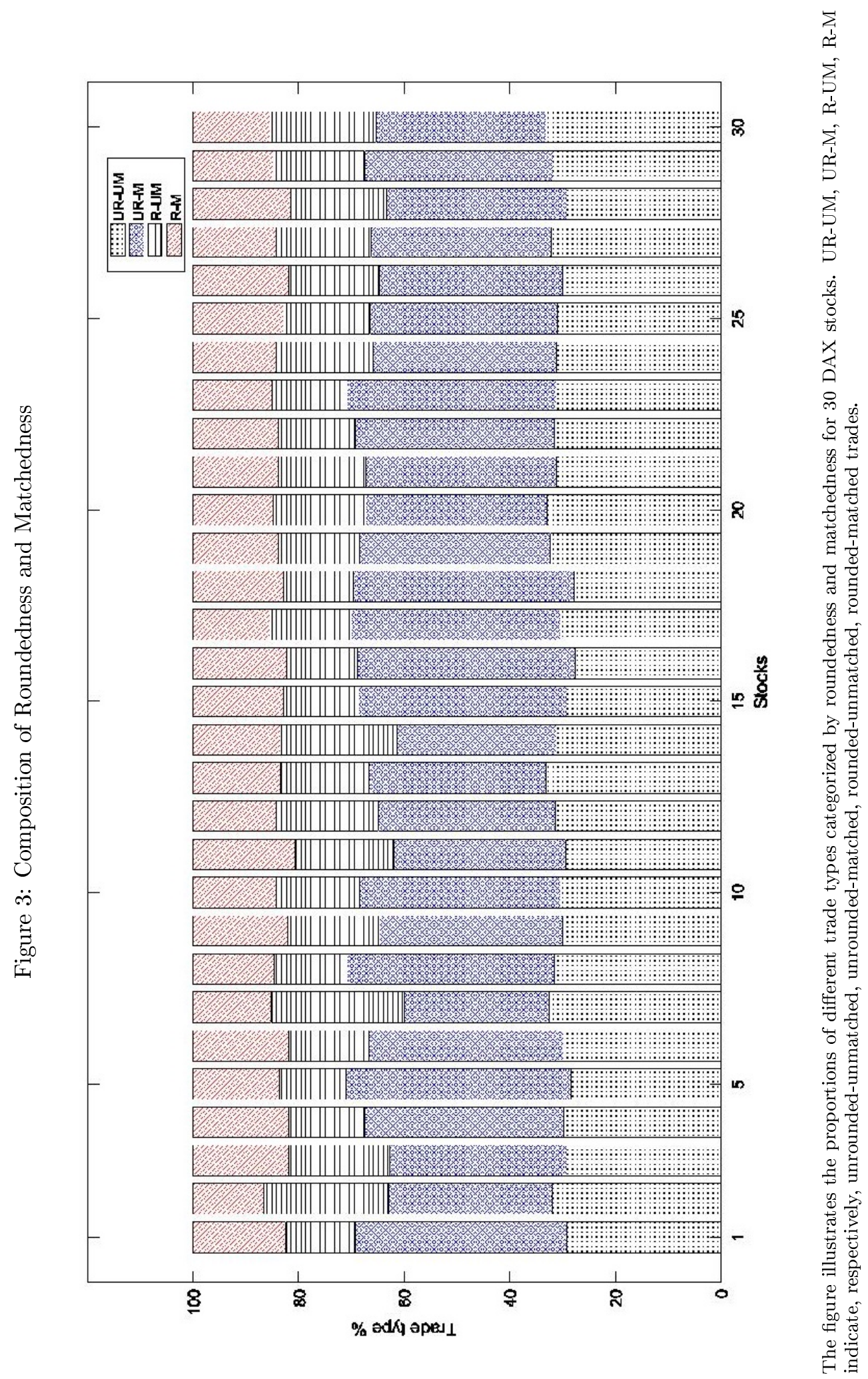




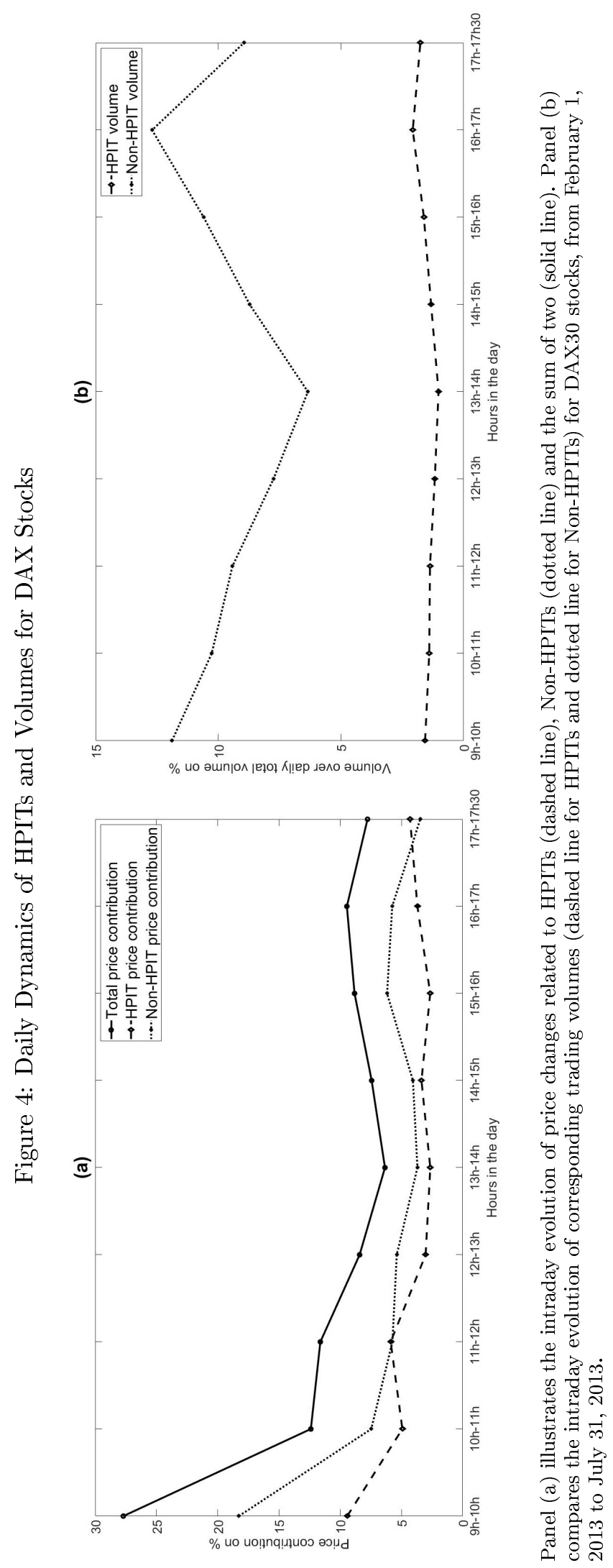


Figure 5: Average Intraday Dynamics of Spread for DAX, MDAX and SDAX Stocks
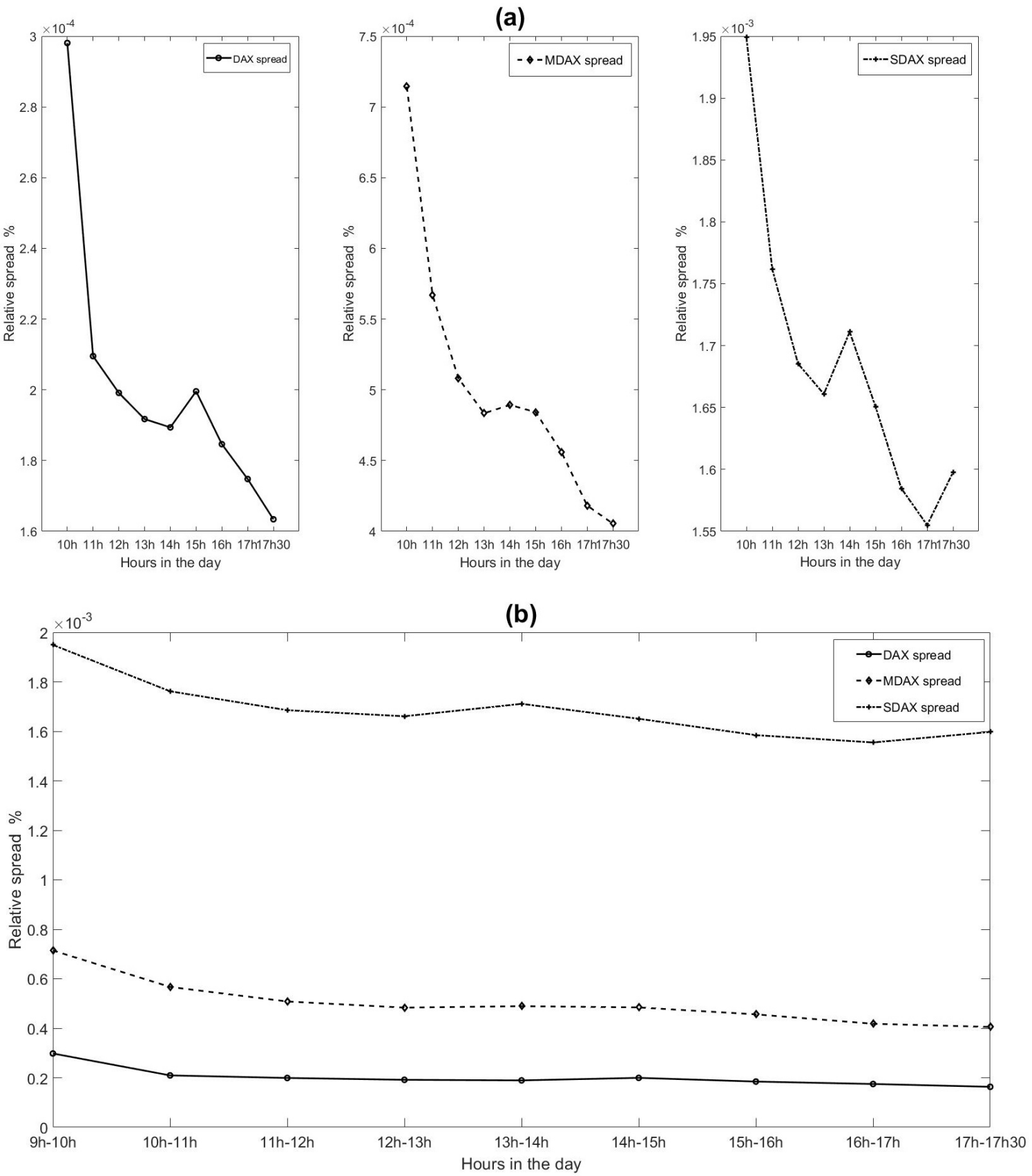

Panel (a) illustrates separately the intraday evolution of average relative bid-ask spread for DAX (solid lines), MDAX (dashed lines), and SDAX (dotted lines) stocks. Panel (b) compares the intraday evolution of average relative bid-ask spread for DAX (solid lines), MDAX (dashed lines), and SDAX (dotted lines) stocks. The sample period covers 6 months from February 1, 2013 to July 31, 2013. Relative bid-ask spread is defined as the ratio of bid-ask spread to midquote price. 
Table 1: Summary Statistics for DAX, MDAX, and SDAX Stocks

\begin{tabular}{llll}
\hline & DAX & MDAX & SDAX \\
\hline A. Sample & & & \\
Number of days & 125 & 125 & 125 \\
Number of stocks & 30 & 50 & 50 \\
B. Daily market & & & \\
$\quad$ Avg. Market Capitalization (in billion Euros) & & & \\
$\quad$ Mean & 3362.67 & 464.00 & 60.29 \\
$\quad$ Median & 2318.76 & 279.04 & 45.32 \\
$\quad$ Standard deviation & 2647.32 & 604.67 & 50.62 \\
Avg.Daily Price (in Euros) & & & \\
$\quad$ Mean & 62.81 & 48.22 & 27.60 \\
Median & 57.48 & 34.09 & 16.44 \\
$\quad$ Standard deviation & 43.07 & 45.54 & 41.74 \\
Avg.Daily Trading Volume (in million shares) & & & \\
Mean & 4.08 & 0.35 & 0.21 \\
Median & 2.09 & 0.19 & 0.03 \\
$\quad$ Standard deviation & 6.71 & 0.52 & 0.58 \\
Avg. Daily Turnover (in percentage) & & & \\
$\quad$ Mean & $0.50 \%$ & $0.31 \%$ & $0.25 \%$ \\
Median & $0.41 \%$ & $0.26 \%$ & $0.16 \%$ \\
$\quad$ Standard deviation & $0.29 \%$ & $0.17 \%$ & $0.38 \%$ \\
Avg. Daily Return (in percentage) & & & \\
Mean & $0.04 \%$ & $0.03 \%$ & $-0.07 \%$ \\
Median & $0.03 \%$ & $0.02 \%$ & $-0.03 \%$ \\
Standard deviation & $0.24 \%$ & $0.13 \%$ & $0.48 \%$ \\
\hline
\end{tabular}

This table reports the statistics for the average market capitalization (in billion Euros), the average daily price (in Euros), the average daily trading volume (in million shares), the average daily turnover (in percentage) defined as the trading volume over the outstanding shares and the average daily (log) return for the stocks in DAX, MDAX and SDAX indexes, from February 1, 2013 to July 31, 2013. All data are from the Compustat Global Security Daily files and based on the primary issues. 
Table 2: Trade and Information Environment Statistics for DAX, MDAX, and SDAX Stocks

\begin{tabular}{|c|c|c|c|}
\hline & DAX & MDAX & SDAX \\
\hline \multicolumn{4}{|l|}{ A. Sample } \\
\hline Number of days & 125 & 125 & 125 \\
\hline Number of stocks & 30 & 50 & 50 \\
\hline \multicolumn{4}{|l|}{ B. Trade environment } \\
\hline \multicolumn{4}{|c|}{ Best bid-ask spread (basis points) } \\
\hline Mean & $5.45 \mathrm{E}-04$ & $1.57 \mathrm{E}-03$ & $5.20 \mathrm{E}-03$ \\
\hline Median & $6.02 \mathrm{E}-04$ & $1.43 \mathrm{E}-03$ & $4.68 \mathrm{E}-03$ \\
\hline Standard deviation & $1.49 \mathrm{E}-04$ & $6.11 \mathrm{E}-04$ & $2.45 \mathrm{E}-03$ \\
\hline \multicolumn{4}{|c|}{ LOB depth ask (cum.5-level) } \\
\hline Mean & 11508 & 1388 & 2667 \\
\hline Median & 3405 & 925 & 1544 \\
\hline Standard deviation & 27368 & 1204 & 4770 \\
\hline \multicolumn{4}{|c|}{ LOB depth bid (cum.5-level) } \\
\hline Mean & 11873 & 1338 & 2854 \\
\hline Median & 3390 & 913 & 1207 \\
\hline Standard deviation & 29515 & 1107 & 5818 \\
\hline \multicolumn{4}{|l|}{ Shares/trade } \\
\hline Mean & 668 & 209 & 481 \\
\hline Median & 259 & 144 & 251 \\
\hline Standard deviation & 1138 & 182 & 744 \\
\hline \multicolumn{4}{|l|}{ Volumes $(€) /$ trade } \\
\hline Mean & 17283.21 & 5982.99 & 4074.04 \\
\hline Median & 15142.64 & 5467.92 & 3972.78 \\
\hline Standard deviation & 6219.74 & 2054.80 & 1042.54 \\
\hline \multicolumn{4}{|c|}{ Duration (second)/per trade } \\
\hline Mean & 9.40 & 46.37 & 268.03 \\
\hline Median & 8.87 & 35.49 & 294.24 \\
\hline Standard deviation & 4.57 & 28.88 & 108.74 \\
\hline \multicolumn{4}{|l|}{ Daily number of trades } \\
\hline Mean & 4527 & 1025 & 185 \\
\hline Median & 3954 & 1012 & 125 \\
\hline Standard deviation & 2149 & 479 & 143 \\
\hline \multicolumn{4}{|c|}{ C. Information environment } \\
\hline \multicolumn{4}{|c|}{ Monthly Number of news per stock in average } \\
\hline Mean & 606 & 104 & 21 \\
\hline Median & 236 & 41 & 16 \\
\hline Standard deviation & 752 & 288 & 14 \\
\hline \multicolumn{4}{|l|}{ Number of analysts } \\
\hline Mean & 29.77 & 2.88 & 2.02 \\
\hline Median & 30 & 3 & 2 \\
\hline Standard deviation & 4.55 & 0.45 & 0.51 \\
\hline \multicolumn{4}{|l|}{ Forecast dispersion } \\
\hline Mean & 0.008 & 0.009 & 0.032 \\
\hline Median & 0.005 & 0.005 & 0.006 \\
\hline Standard deviation & 0.015 & 0.014 & 0.146 \\
\hline \multicolumn{4}{|l|}{ Forecast error } \\
\hline Mean & 0.007 & 0.013 & 0.055 \\
\hline Median & 0.002 & 0.004 & 0.005 \\
\hline Standard deviation & 0.015 & 0.023 & 0.226 \\
\hline
\end{tabular}

This table reports the statistics for trading and information environment variables. The best bid-ask spread is the relative bid-ask spread on percentage. LOB depth ask (bid) is the cumulative quantity available for the first three levels at the ask (bid) side of the LOB. Duration/trade is the time between two consecutive trades. The monthly number of news is the number of times that the company is mentioned in the mass media and the news data are from the RavenPack dataset. Finally, trades hit by hidden orders is the proportion of market orders that are matched with iceberg or hidden orders embedded in the open LOB. 


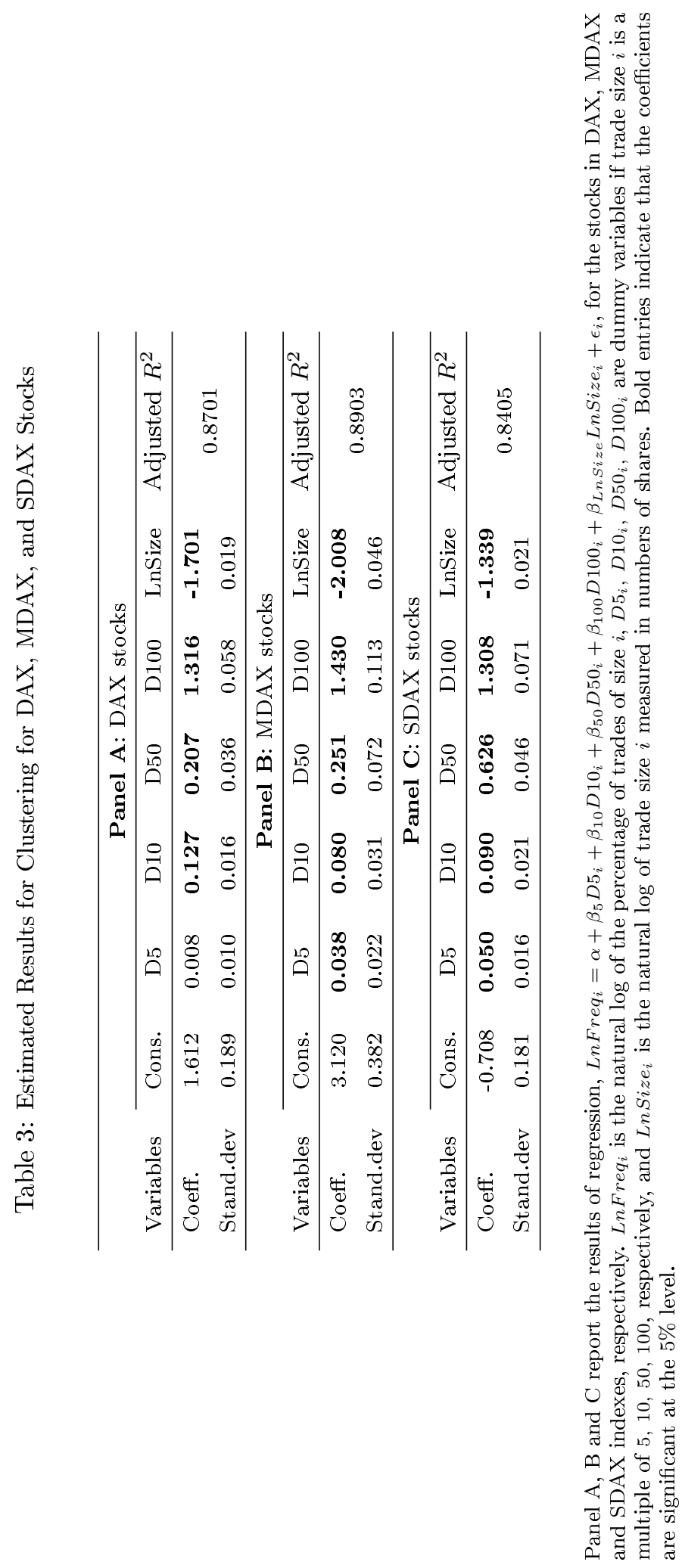




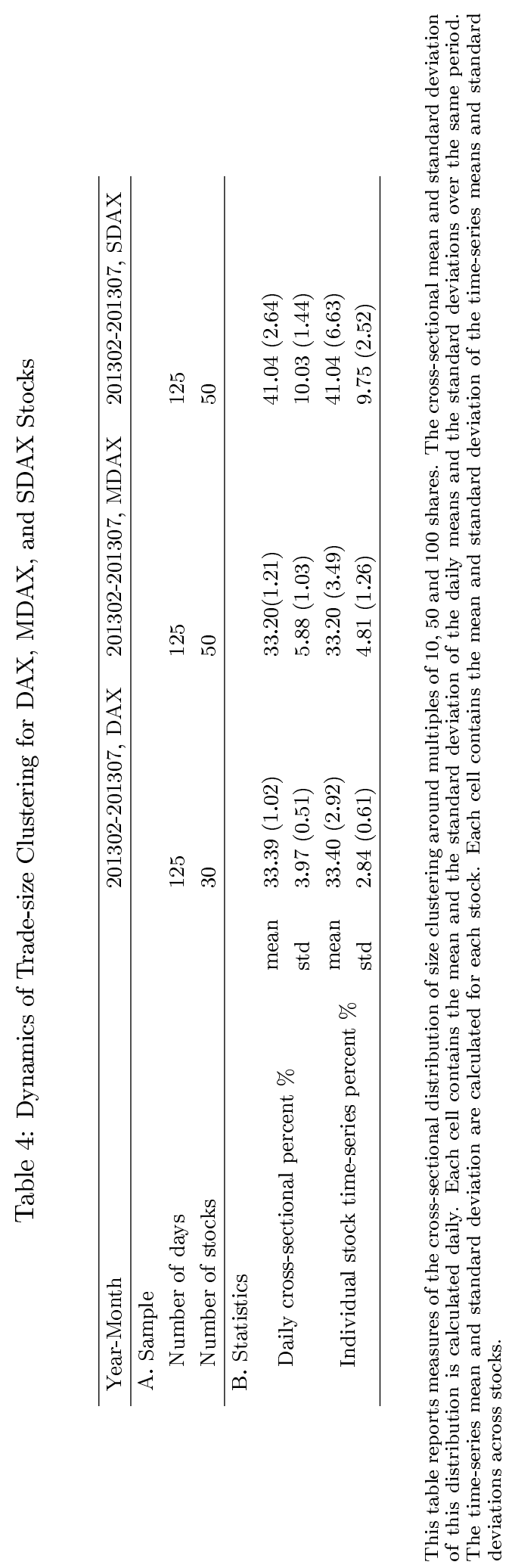




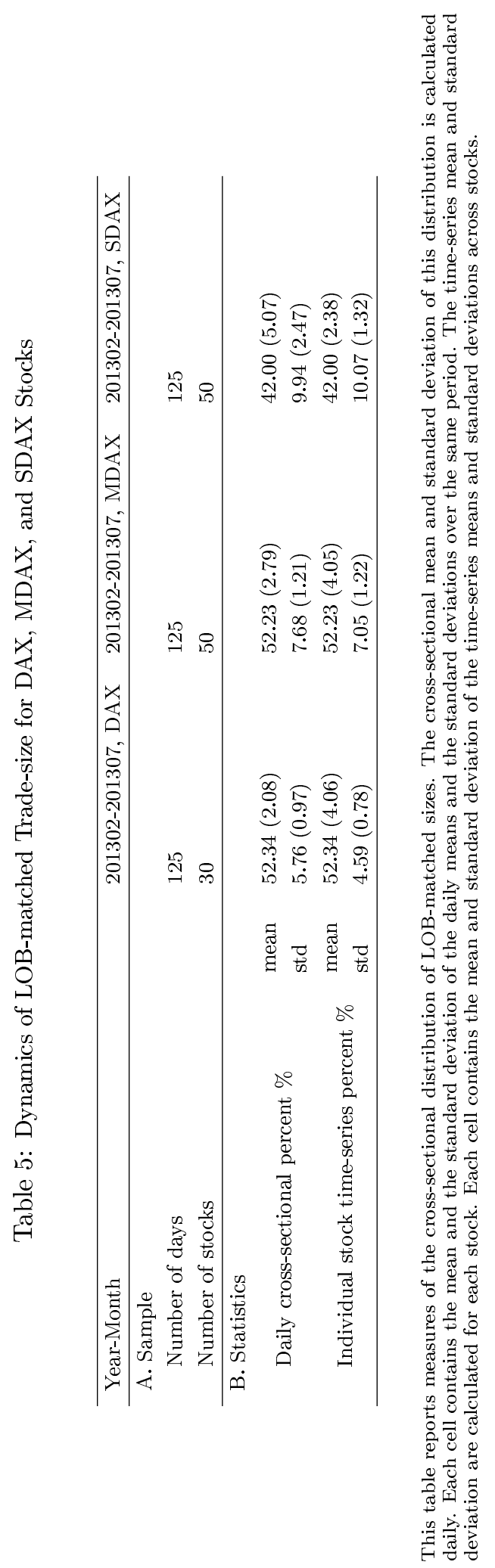




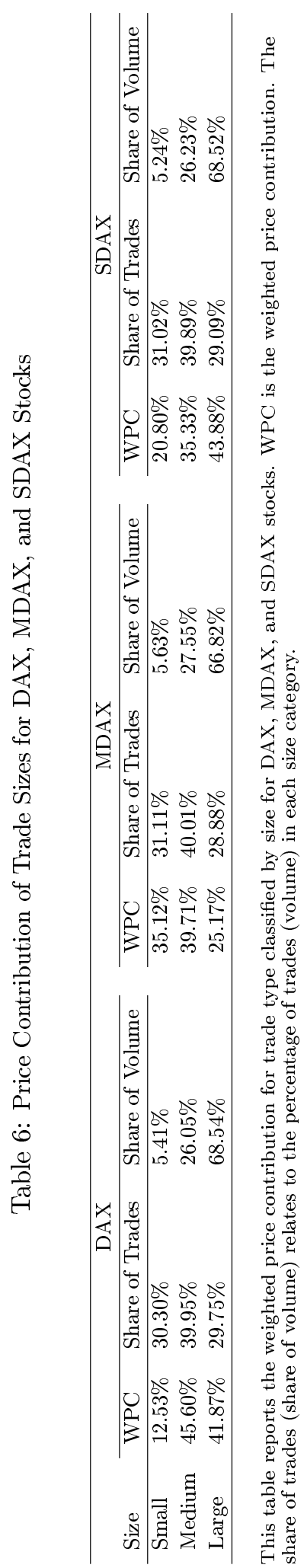




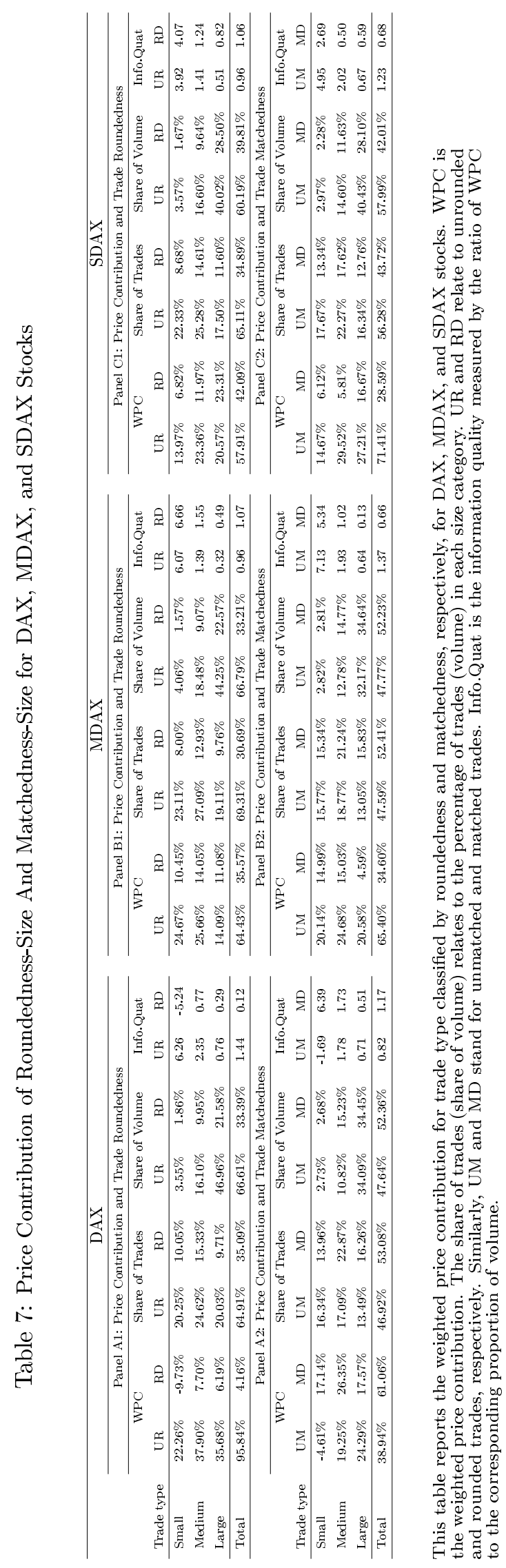




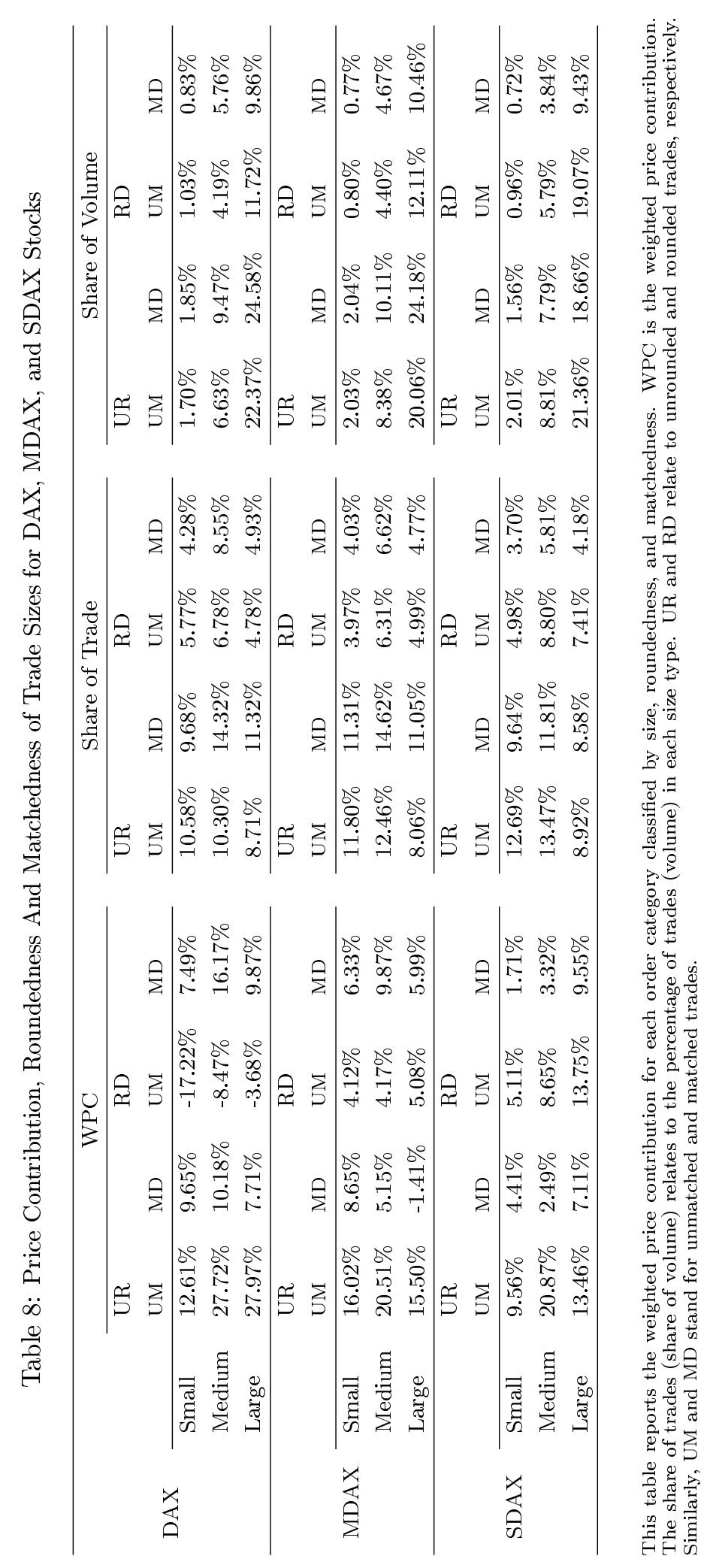


Table 9: Regression Results of Price Discovery and Roundedness and Matchedness of Trade Sizes for DAX, MDAX, and SDAX

\begin{tabular}{|c|c|c|c|c|c|c|c|c|}
\hline & \multirow[b]{2}{*}{ Trade types } & & \multicolumn{2}{|l|}{ DAX } & \multicolumn{2}{|l|}{ MDAX } & \multicolumn{2}{|l|}{ SDAX } \\
\hline & & & Param. & $\mathrm{P}$-value & Param. & P-value & Param. & $\mathrm{P}$-value \\
\hline \multirow[t]{4}{*}{ Small } & Unrounded & Unmatched & 0.004 & $<0.01$ & 0.003 & $<0.01$ & 0.003 & $<0.01$ \\
\hline & & Matched & 0.003 & $<0.01$ & 0.001 & $<0.01$ & 0.001 & 0.036 \\
\hline & Rounded & Unmatched & -0.006 & $<0.01$ & 0.001 & $<0.01$ & 0.001 & 0.028 \\
\hline & & Matched & 0.002 & $<0.01$ & 0.001 & $<0.01$ & $3.47 \mathrm{E}-04$ & 0.384 \\
\hline \multirow[t]{4}{*}{ Medium } & Unrounded & Unmatched & 0.008 & $<0.01$ & 0.003 & $<0.01$ & 0.004 & $<0.01$ \\
\hline & & Matched & 0.002 & 0.336 & -0.001 & 0.063 & -0.002 & 0.014 \\
\hline & Rounded & Unmatched & -0.003 & $<0.01$ & 0.000 & 0.401 & 0.001 & 0.350 \\
\hline & & Matched & 0.005 & $<0.01$ & 0.001 & $<0.01$ & $-2.61 \mathrm{E}-04$ & 0.662 \\
\hline \multirow[t]{4}{*}{ Large } & Unrounded & Unmatched & 0.006 & 0.150 & $1.06 \mathrm{E}-05$ & 0.976 & -0.003 & 0.098 \\
\hline & & Matched & -0.001 & 0.848 & -0.004 & $<0.01$ & -0.005 & $<0.01$ \\
\hline & Rounded & Unmatched & -0.003 & 0.126 & -0.001 & $<0.01$ & -0.002 & 0.014 \\
\hline & & Matched & 0.002 & 0.381 & $-4.10 \mathrm{E}-04$ & 0.049 & $-1.62 \mathrm{E}-04$ & 0.845 \\
\hline Constant & & & 0.014 & 0.443 & 0.015 & $<0.01$ & 0.038 & $<0.01$ \\
\hline Adjusted $R^{2}$ & & & 0.0091 & & 0.0076 & & 0.0049 & \\
\hline Obs & & & 44,904 & & 74,856 & & 41,940 & \\
\hline
\end{tabular}

This table reports the results of weighted least square regressions of WPC on the percentage of the transaction (volume) and dummies based on roundedness, matchedness, and size, $C u m P C_{j}^{s, t}=\sum_{j=1}^{k} \alpha_{j} \times d u m m y_{j}+\beta \times$ PcntVolume $e_{j}^{s, t}+\epsilon_{j}^{s, t}$, for DAX, MDAX, and SDAX stocks. Bold entries indicate that the coefficients are significant at the $5 \%$ level. Obs is the number of observations in the regression. From the sample, we exclude the days that have the same open and close prices. 
Table 10: The Informational Quality of HPITs for DAX, MDAX and SDAX

\begin{tabular}{llll}
\hline & \multicolumn{3}{c}{ Panel a: } \\
\cline { 2 - 4 } & WPC & Trade $\%$ & Volume $\%$ \\
\hline DAX & $73.6 \%$ & $43.4 \%$ & $16.8 \%$ \\
MDAX & $65.5 \%$ & $50.2 \%$ & $18.7 \%$ \\
SDAX & $40.0 \%$ & $40.8 \%$ & $13.3 \%$ \\
\hline
\end{tabular}

\begin{tabular}{|c|c|c|}
\hline \multicolumn{3}{|c|}{ Panel b: Informational Quality of the HPITs } \\
\hline & WPC/Trade\% & WPC/Volume\% \\
\hline DAX & 1.697 & 4.392 \\
\hline MDAX & 1.305 & 3.508 \\
\hline SDAX & 0.980 & 2.997 \\
\hline
\end{tabular}

Panel (a) reports the weighted price contribution (WPC) of HPITs for DAX, MDAX and SDAX stocks based on the regression, CumPC $C_{j}^{s, t}=\sum_{j=1}^{k} \alpha_{j} \times d u m m y_{j}+\beta \times$ PcntVolume $_{j}^{s, t}+\epsilon_{j}^{s, t}$. Trade $\%$ is the ratio of HPITs numbers over total trade numbers, and volume\% is the ratio of HPITs volumes over total trade volumes. Panel (b) represents the corresponding informational qualities, measured by WPC/Trade\% and WPC/Volume\%. 


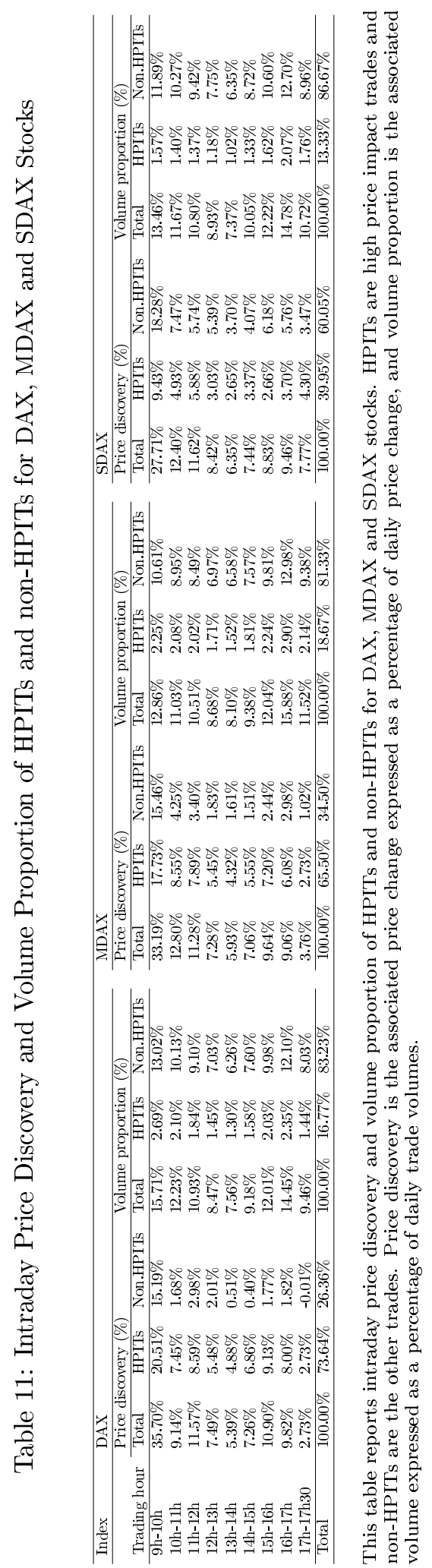


Table 12: The Effect of HPITs of DAX Stocks on 15-min Conditional Volatility

\begin{tabular}{|c|c|c|c|c|c|c|c|}
\hline Param & $\omega$ & $a$ & $\vartheta$ & $\beta$ & $\gamma$ & $\mathrm{Q}(15)$ & Q2(15) \\
\hline ADS & $-1.913 * * *$ & -0.003 & $0.259 * * *$ & $0.844 * * *$ & $-2.038 * * *$ & 19.44 & 4.74 \\
\hline $\mathrm{ALV}$ & $-2.002 * * *$ & $-0.045 * * *$ & $0.226 * * *$ & $0.836 * * *$ & $-1.304 * * *$ & 18.44 & 11.76 \\
\hline BAS & $-1.900 * * *$ & $-0.025^{* * *}$ & $0.168 * * *$ & $0.845^{* * *}$ & $-2.703 * * *$ & 29.02 & 12.66 \\
\hline BAYN & $-2.356 * * *$ & $-0.017 * *$ & $0.216^{* * *}$ & $0.807 * * *$ & $-1.753 * * *$ & 17.84 & 5.99 \\
\hline BEI & $-4.302 * * *$ & $0.018^{*}$ & $0.360 * * *$ & $0.657 * * *$ & $-0.616^{* * *}$ & 7.74 & 5.31 \\
\hline BMW & $-0.113 * * *$ & $-0.011 * * *$ & $0.051^{* * *}$ & $0.990 * * *$ & $-3.372 * * *$ & 11.22 & 17.98 \\
\hline CBK & $-0.696 * * *$ & $-0.041 * * *$ & $0.204^{* * *}$ & $0.935^{* * *}$ & $-4.686 * * *$ & 14.64 & 71.82 \\
\hline $\mathrm{CON}$ & $-2.370 * * *$ & -0.003 & $0.291 * * *$ & $0.800 * * *$ & $-1.856 * * *$ & 18.39 & 11.54 \\
\hline DAI & $-2.081 * * *$ & -0.011 & $0.255^{* * *}$ & $0.824 * * *$ & $-2.936 * * *$ & 12.62 & 6.70 \\
\hline DB1 & $-0.999 * * *$ & $0.083 * * *$ & $0.246 * * *$ & $0.915 * * *$ & $-3.134 * * *$ & 16.61 & 2.19 \\
\hline DBK & $-1.197 * * *$ & $-0.034 * * *$ & $0.167 * * *$ & $0.896 * * *$ & $-4.077 * * *$ & 14.32 & 14.07 \\
\hline DPW & $-1.998 * * *$ & -0.013 & $0.314^{* * *}$ & $0.840 * * *$ & $-0.674 * * *$ & 14.10 & 9.60 \\
\hline DTE & $-0.907 * * *$ & $0.025^{* * *}$ & $0.181^{* * *}$ & $0.927^{* * *}$ & $-3.290 * * *$ & 17.74 & 8.50 \\
\hline EOAN & $-0.862 * * *$ & -0.006 & $0.201 * * *$ & $0.928 * * *$ & $-2.202 * * *$ & 24.65 & 9.59 \\
\hline FME & $-1.231 * * *$ & 0.007 & $0.277 * * *$ & $0.900 * * *$ & $-1.026 * * *$ & 27.20 & 30.15 \\
\hline FRE & $-1.896 * * *$ & -0.011 & $0.249 * * *$ & $0.847 * * *$ & 0.042 & 21.28 & 2.74 \\
\hline HEI & $-1.995 * * *$ & 0.004 & $0.262 * * *$ & $0.831 * * *$ & $-1.139 * * *$ & 23.82 & 3.95 \\
\hline HEN 3 & $-2.813^{* * *}$ & $0.025^{* *}$ & $0.220 * * *$ & $0.773 * * *$ & $-0.526 * * *$ & 17.33 & 6.47 \\
\hline IFX & $-1.241 * * *$ & $-0.016^{*}$ & $0.262 * * *$ & $0.894 * * *$ & $-2.893^{* * *}$ & 12.09 & 13.43 \\
\hline LHA & $-2.668 * * *$ & -0.009 & $0.378 * * *$ & $0.772^{* * *}$ & $-1.817 * * *$ & 12.11 & 7.08 \\
\hline LIN & $-2.146 * * *$ & $-0.053 * * *$ & $0.167 * * *$ & $0.831 * * *$ & $-1.201 * * *$ & 28.36 & 12.72 \\
\hline LXS & $-2.338 * * *$ & $-0.023 * * *$ & $0.313^{* * *}$ & $0.800 * * *$ & $-3.273 * * *$ & 21.53 & 8.09 \\
\hline MRK & $-2.213 * * *$ & 0.005 & $0.253 * * *$ & $0.821^{* * *}$ & $-1.225^{* * *}$ & 10.92 & 5.29 \\
\hline MUV2 & $-1.404 * * *$ & -0.014 & $0.237 * * *$ & $0.886 * * *$ & $-1.587 * * *$ & 15.93 & 11.83 \\
\hline RWE & $-0.101 * * *$ & 0.000 & $0.080 * * *$ & $0.991 * * *$ & $-3.750 * * *$ & 16.02 & 21.43 \\
\hline SAP & $-0.052 * * *$ & $-0.015^{* * *}$ & $0.039 * * *$ & $0.996^{* * *}$ & $-2.682 * * *$ & 14.97 & 44.75 \\
\hline $\mathrm{SDF}$ & $-0.154 * * *$ & $-0.033 * * *$ & $0.129 * * *$ & $0.986 * * *$ & $-3.136 * * *$ & 14.90 & 89.48 \\
\hline SIE & $-0.282 * * *$ & $-0.039 * * *$ & $0.099 * * *$ & $0.977 * * *$ & $-4.240 * * *$ & 32.80 & 5.47 \\
\hline TKA & $-3.460 * * *$ & $0.030 * * *$ & $0.459 * * *$ & $0.697 * * *$ & $-1.886^{* * *}$ & 17.01 & 4.04 \\
\hline VOW3 & $-2.295 * * *$ & $-0.117 * * *$ & $0.413 * * *$ & $0.805^{* * *}$ & $-2.265 * * *$ & 12.06 & 4.27 \\
\hline Mean & -1.67 & -0.01 & 0.23 & 0.86 & -2.24 & 17.84 & 15.45 \\
\hline Min & -4.30 & -0.12 & 0.05 & 0.66 & -4.69 & 7.74 & 2.19 \\
\hline Max & -0.05 & 0.08 & 0.16 & 1.00 & 0.04 & 32.80 & 89.48 \\
\hline
\end{tabular}

This table reports the estimated results of the EGARCH model, $\log \left(\sigma_{i}^{2}\right)=\omega+\sum_{j=1}^{p} \alpha_{j} g\left(Z_{i-j}\right)+\sum_{j=1}^{q} \beta_{j} \log \left(\sigma_{i-j}^{2}\right)+$ $\gamma H P I T_{\mathrm{i}-1} \%$, for 15 -min deseasonalized returns for DAX stocks. The results remain qualitatively similar for the 30-min interval. Q(15) and Q2(15) relate to Ljung-Box statistics on 15 lagged standardized residuals and squared standardized residuals derived from the model. The $5 \%$ critical value is 24.99 . ***, ** and * denote either coefficient estimates that are significantly different from zero or test statistics that are significant at the $1 \%, 5 \%$, and $10 \%$, respectively. 


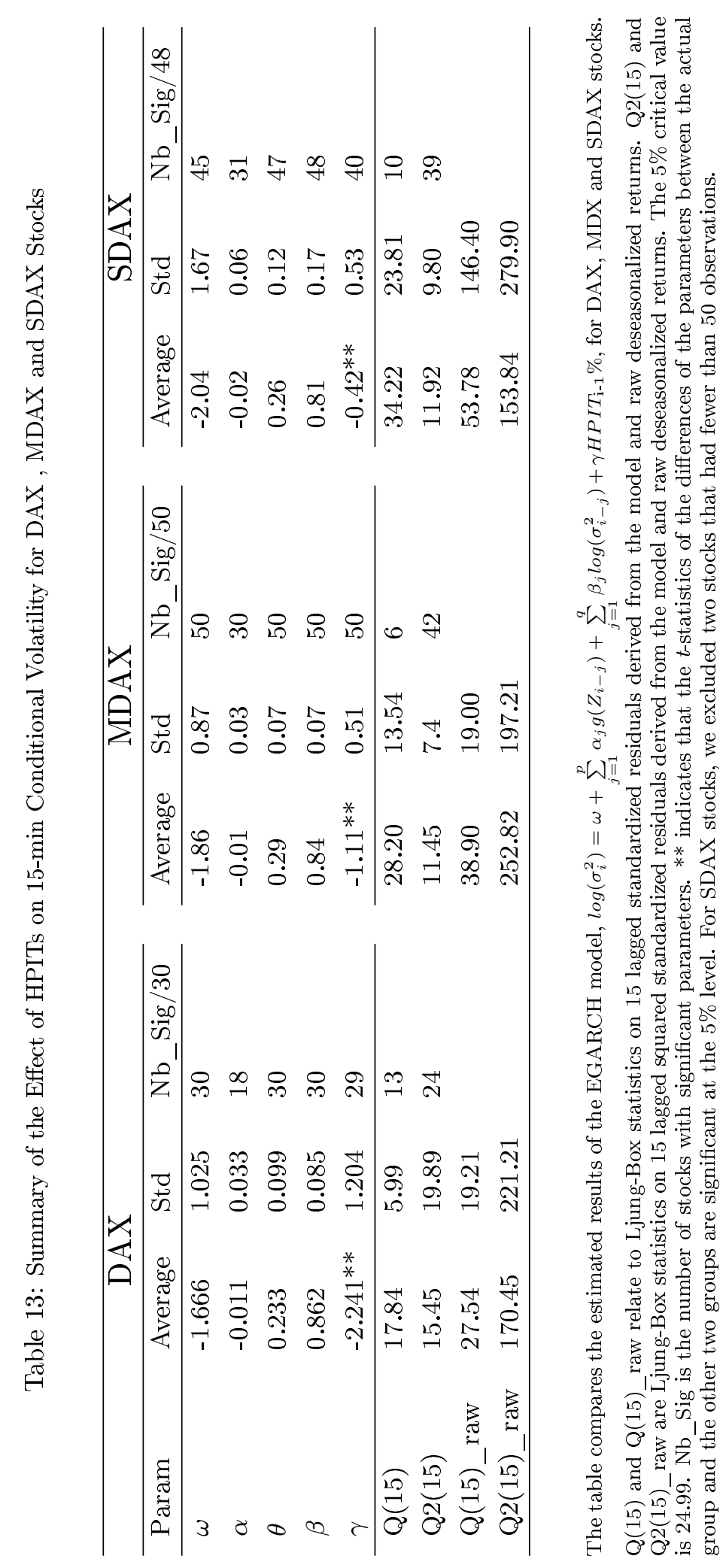


Table 14: Effect of HPITs on Autocorrelation for DAX Stocks

\begin{tabular}{|c|c|c|c|c|c|c|c|c|c|c|}
\hline & $\delta_{i, 1}$ & $\delta_{i, 2}$ & $\delta_{i, 3}$ & $\delta_{i, 4}$ & $\psi_{i, 1}$ & $\psi_{i, 2}$ & $\psi_{i, 3}$ & $\psi_{i, 4}$ & $\delta_{i, 1}+\delta_{i, 2}$ & Adjusted $R^{2}$ \\
\hline ADS & $-0.7965^{* * *}$ & -0.2587 & $0.0249^{* * *}$ & -0.0106 & 0.0004 & -0.0150 & $0.0011^{* * *}$ & -0.0008 & $-1.0552^{* * *}$ & 0.145 \\
\hline ALV & $-1.1133^{* * *}$ & -0.0812 & -0.0106 & 0.0290 & $-0.0016^{* * *}$ & -0.0090 & $0.0014^{* * *}$ & 0.0008 & $-1.1945^{* * *}$ & 0.244 \\
\hline BAS & $-1.0273^{* * *}$ & -0.0052 & 0.0118 & -0.0397 & $-0.0021^{* *}$ & 0.0316 & $0.0010^{* * *}$ & 0.0014 & $-1.0325^{* * *}$ & 0.140 \\
\hline BAYN & $-0.6011^{* * *}$ & 0.1881 & $0.0821^{* * *}$ & $0.0850^{* *}$ & 0.0005 & 0.0012 & $0.0009^{* * *}$ & -0.0020 & $-0.4130^{*}$ & 0.121 \\
\hline BEI & $-0.9144^{* * *}$ & -0.0126 & 0.0034 & -0.0221 & 0.0019 & -0.0206 & $0.0010^{* * *}$ & 0.0004 & $-0.9270^{* * *}$ & 0.144 \\
\hline BMW & $-1.1628^{* * *}$ & -0.0575 & -0.0121 & $-0.0471^{* *}$ & -0.0009 & 0.0380 & $0.0009^{* * *}$ & 0.0005 & $-1.2203^{* * *}$ & 0.093 \\
\hline CBK & $-0.5340^{* * *}$ & $0.6621^{* * *}$ & 0.0086 & 0.0249 & 0.0022 & 0.1569 & $0.0038^{* * *}$ & -0.0003 & 0.1281 & 0.360 \\
\hline $\mathrm{CON}$ & $-0.8454^{* * *}$ & -0.1152 & 0.0260 & 0.0264 & 0.0004 & -0.0029 & $0.0012^{* * *}$ & 0.0007 & $-0.9605^{* * *}$ & 0.124 \\
\hline DAI & $-1.1257^{* * *}$ & -0.2624 & 0.0050 & $-0.0821^{*}$ & 0.0005 & -0.0170 & $0.0016^{* * *}$ & 0.0005 & $-1.3881^{* * *}$ & 0.184 \\
\hline DB1 & $-0.7204^{* * *}$ & 0.0608 & -0.0022 & -0.0160 & 0.0048 & $-0.0586^{* *}$ & $0.0012^{* * *}$ & 0.0027 & $-0.6596^{* *}$ & 0.169 \\
\hline DBK & $-1.1506^{* * *}$ & -0.2265 & 0.0283 & -0.0758 & $-0.0036^{* *}$ & 0.0364 & $0.0020^{* * *}$ & 0.0007 & $-1.3771^{* * *}$ & 0.249 \\
\hline DPW & $-1.1992^{* * *}$ & -0.0518 & $0.0545^{* * *}$ & 0.0106 & -0.0018 & -0.0637 & $0.0010^{* * *}$ & 0.0016 & $-1.2510^{* * *}$ & 0.177 \\
\hline DTE & $-1.3341^{* * *}$ & -0.2429 & 0.0378 & -0.0022 & -0.0009 & -0.0116 & $0.0011^{* * *}$ & 0.0012 & $-1.5770^{* * *}$ & 0.197 \\
\hline EOAN & $-0.8597^{* * *}$ & $-0.3674^{* *}$ & 0.0065 & 0.0285 & $-0.0120^{* *}$ & -0.1999 & $0.0016^{* * *}$ & -0.0007 & $-1.2271^{* * *}$ & 0.272 \\
\hline FME & $-1.1554^{* * *}$ & -0.1251 & 0.0021 & -0.0176 & -0.0018 & 0.0207 & $0.0009 * * *$ & 0.0001 & $-1.2805^{* * *}$ & 0.135 \\
\hline FRE & $-0.5119^{* * *}$ & 0.0809 & $0.0540^{* *}$ & -0.0244 & -0.0011 & -0.0071 & $0.0008^{* * *}$ & -0.0018 & $-0.4309^{* *}$ & 0.115 \\
\hline HEI & $-1.1860 * * *$ & $0.3942^{* *}$ & $0.0502^{*}$ & 0.0325 & -0.0004 & -0.0256 & $0.0008^{* * *}$ & $0.0029^{*}$ & $-0.7918^{* * *}$ & 0.112 \\
\hline HEN3 & $-0.8704^{* * *}$ & 0.2582 & -0.0190 & $0.0533^{* *}$ & 0.0005 & $-0.0265^{*}$ & $0.0008^{* * *}$ & 0.0004 & $-0.6122^{* * *}$ & 0.093 \\
\hline IFX & $-1.0782^{* * *}$ & -0.0730 & $0.0867^{* * *}$ & 0.0045 & -0.0134 & -0.1552 & $0.0014^{* * *}$ & 0.0015 & $-1.1512^{* * *}$ & 0.177 \\
\hline LHA & $-0.5453^{* * *}$ & 0.1934 & 0.0454 & -0.0102 & -0.0046 & $-0.1954^{* *}$ & $0.0015^{* * *}$ & -0.0007 & -0.3519 & 0.160 \\
\hline LIN & $-0.7941^{* * *}$ & $-0.5138^{* *}$ & 0.0321 & 0.0213 & $-0.0009^{* *}$ & -0.0142 & $0.0009^{* * *}$ & 0.0011 & $-1.3079^{* * *}$ & 0.160 \\
\hline LXS & $-0.3159^{* *}$ & -0.0278 & $0.0628^{* *}$ & 0.0043 & 0.0019 & -0.0024 & $0.0018^{* * *}$ & 0.0004 & $-0.3437^{*}$ & 0.209 \\
\hline MRK & $-0.6054^{* * *}$ & $0.5134^{* * *}$ & $0.0754^{* * *}$ & 0.0103 & -0.0002 & -0.0079 & $0.0010^{* * *}$ & -0.0011 & -0.0920 & 0.189 \\
\hline MUV2 & $-0.8635^{* * *}$ & -0.2252 & $0.0662^{* *}$ & $0.0724^{* * *}$ & -0.0006 & 0.0029 & $0.0012^{* * *}$ & 0.0010 & $-1.0887^{* * *}$ & 0.189 \\
\hline RWE & $-0.9280 * * *$ & $-0.5442^{* * *}$ & 0.0067 & 0.0036 & -0.0034 & $-0.1124^{*}$ & $0.0014^{* * *}$ & $0.0024 * * *$ & $-1.4722^{* * *}$ & 0.184 \\
\hline SAP & $-1.2399 * * *$ & -0.2036 & -0.0302 & -0.0325 & 0.0012 & -0.0219 & $0.0008^{* * *}$ & -0.0006 & $-1.4434^{* * *}$ & 0.108 \\
\hline SDF & $-0.9489 * * *$ & -0.0649 & 0.0053 & $-0.0383^{* *}$ & $-0.0145^{* * *}$ & 0.0464 & $0.0014^{* * *}$ & $0.0016^{*}$ & $-1.0138^{* * *}$ & 0.348 \\
\hline SIE & $-1.0153^{* * *}$ & -0.2589 & -0.0175 & -0.0366 & -0.0024 & -0.0255 & $0.0011^{* * *}$ & -0.0017 & $-1.2742^{* * *}$ & 0.195 \\
\hline TKA & $-0.5232^{* * *}$ & -0.2820 & 0.0016 & 0.0389 & -0.0007 & 0.0042 & $0.0021^{* * *}$ & 0.0009 & $-0.8052^{* * *}$ & 0.207 \\
\hline VOW3 & $-1.1286^{* * *}$ & $-0.3820^{*}$ & 0.0148 & -0.0211 & -0.0008 & 0.0100 & $0.0018^{* * *}$ & 0.0008 & $-1.5106^{* * *}$ & 0.289 \\
\hline Bonferroni p-value & $<0.001$ & 0.050 & 0.003 & 0.254 & $<0.001$ & 1 & $<0.001$ & 0.186 & $<0.001$ & \\
\hline Nb significant & 30 & 7 & 9 & 6 & 6 & 4 & 30 & 3 & 27 & \\
\hline Mean & -0.9031 & -0.0677 & 0.0234 & -0.0010 & $\begin{array}{c}-0.0018 \\
\end{array}$ & -0.0215 & 0.0013 & 0.0005 & -0.9708 & 0.1829 \\
\hline Median & -0.9212 & -0.0771 & 0.0133 & 0.0007 & -0.0008 & -0.0103 & 0.0011 & 0.0006 & -1.0720 & 0.1768 \\
\hline Min & -1.3341 & -0.5442 & -0.0302 & -0.0821 & -0.0145 & -0.1999 & 0.0008 & -0.0020 & -1.5770 & 0.0932 \\
\hline Max & -0.3159 & 0.6621 & 0.0867 & 0.0850 & 0.0048 & 0.1569 & 0.0038 & 0.0029 & 0.1281 & 0.3596 \\
\hline
\end{tabular}

The table reports the estimated results of the following model:

$R_{i, t}=\left(\delta_{1, i} H_{P I T} \%_{i, \mathrm{t}-1} * 1_{(\text {contrarian })}+\delta_{2, i} H P I T \%_{i, \mathrm{t}-1} * 1_{(\text {herding }}+\delta_{3, i}\right.$ Volume $\left._{t-1}\right) R_{i, t-1}+\psi_{i, 1}$ Range $_{i, t-1}+$ $\psi_{i, 2}$ Spread $_{i, t-1}+\psi_{i, 3}$ Price $_{i, t-1}+\sum_{k=2}^{5} \beta_{i, k} R_{i, t-k}+\sum_{k=1}^{N} \alpha_{i, k} D_{k t}+e_{i, t}$,

for DAX stocks. Bonferroni $p$-value is the $p$-value based on the Bonferroni correction and is calculated as $\min \left(1, \min \left(p_{1}, \ldots, p_{n}\right) \times n\right)$, where $p_{i}$ is the $p$-value of a given parameter from the estimation of the model for the $i$ th stock. A Bonferroni $p$-value less than 0.05 suggests that the null hypothesis is rejected jointly across all stocks at the $5 \%$ significance level or higher. Nb significant is the number of stocks for which the results are statistically significant at the $10 \%$ level. ***,** and $*$ denote either coefficient estimates that are significantly different from zero or test statistics that are significant at the $1 \%, 5 \%$, and $10 \%$ levels, respectively. 


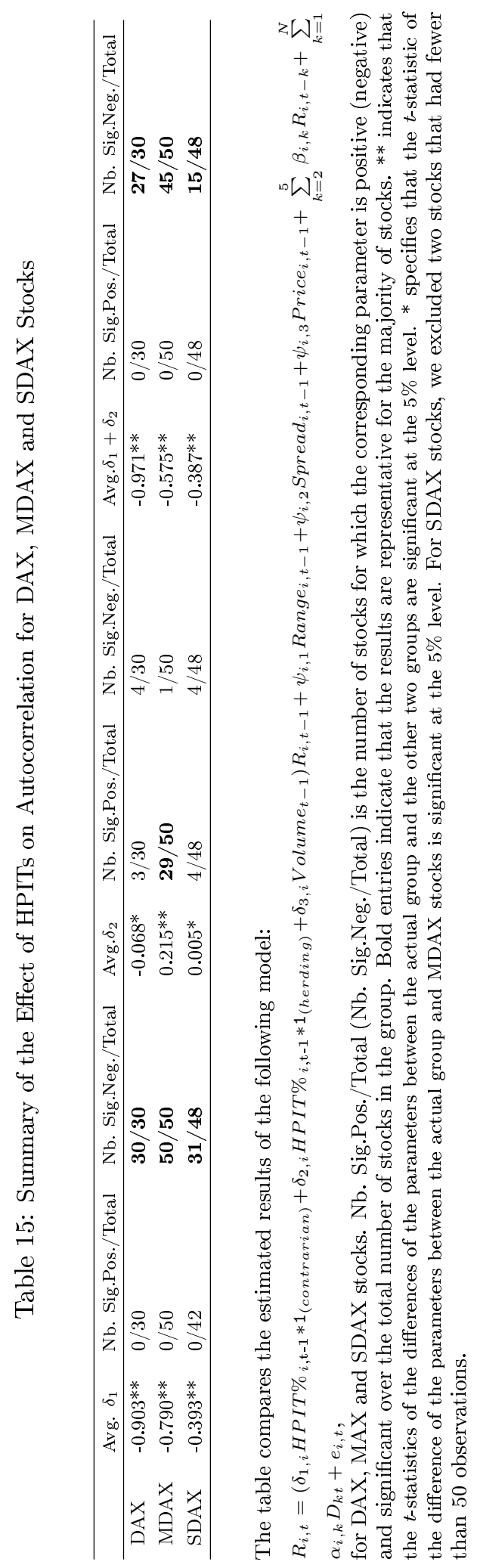


Table 16: The Effect of HPITs on Market Efficiency (Variance Ratio-based Measure)

\begin{tabular}{lllllll}
\hline & \multicolumn{2}{c}{ DAX } & \multicolumn{2}{c}{ MDAX } & \multicolumn{2}{c}{ SDAX } \\
\hline HPIT $_{t-1}$ & $-0.46^{* * *}$ & $-0.36^{* * *}$ & -0.05 & -0.06 & $0.10^{* *}$ & $0.09^{* *}$ \\
& $(-2.75)$ & $(-3.03)$ & $(-0.84)$ & $(-1.23)$ & $(2.13)$ & $(2.45)$ \\
Price $_{t-1}$ & $-0.123^{* * *}$ & 0.014 & -0.037 & 0.013 & -0.020 & 0.004 \\
& $(-2.60)$ & $(1.10)$ & $(-0.80)$ & $(1.60)$ & $(-1.46)$ & $(1.02)$ \\
Range $_{t-1}$ & $-0.034^{* * *}$ & $-0.041^{* * *}$ & -0.010 & $-0.015^{*}$ & $-0.016^{* * *}$ & -0.001 \\
& $(-4.20)$ & $(-3.79)$ & $(-1.16)$ & $(-1.78)$ & $(-3.93)$ & $(-0.54)$ \\
Spread $_{t-1}$ & $-0.091^{* * *}$ & $-0.026^{* * *}$ & $-0.022^{* * *}$ & $-0.009^{* * *}$ & $-4.0 \mathrm{E}-04$ & $1.60 \mathrm{E}-04$ \\
& $(-8.40)$ & $(-4.59)$ & $(-8.91)$ & $(-6.99)$ & $(-0.73)$ & $(0.45)$ \\
Constant & & $0.536^{* * *}$ & & $0.488^{* * *}$ & & $0.371^{* * *}$ \\
& & $(10.85)$ & & $(15.71)$ & & $(21.16)$ \\
\hline Effet & Fixed & Random & Fixed & Random & Fixed & Random \\
Observation & 7484 & 7484 & 12476 & 12476 & 6990 & 6990 \\
Adjusted $R^{2}$ & 0.053 & 0.018 & 0.023 & 0.012 & 0.003 & 0.004 \\
No.tickers & 30 & 30 & 50 & 50 & 49 & 49 \\
\hline
\end{tabular}

The table presents the fix- and random-effect panel regression results on variance-ratio based market price efficiency:

$\left|M_{r}(q)-1\right|_{i, t}=\alpha_{i}+\gamma_{t}+\beta_{1} \times H P I T_{i, t-1}+\beta_{2} \times \log \left(\right.$ Price $\left._{i, t-1}\right)+\beta_{3} \times$ Range $_{i, t-1}+\beta_{4} \times$ Spread $_{i, t-1}+\epsilon_{i, t}$,

$\left|M_{r}(q)-1\right|_{i, t}=\mu+\alpha_{i}+\gamma_{t}+\beta_{1} \times H_{P I T_{i, t-1}}+\beta_{2} \times \log \left(\right.$ Price $\left._{i, t-1}\right)+\beta_{3} \times \operatorname{Range}_{i, t-1}+\beta_{4} \times$ Spread $_{i, t-1}+\epsilon_{i, t}$,

for DAX, MDAX, and SDAX stocks with $4 \mathrm{~h}$ measurement interval. $H P I T_{i, t-1}$ is the the proportion of HPITs for stock $i$ during the period $i-1$, Range $i, t-1$ relates to the range between maximum and minimum price, and Spread $_{i, t-1}$ and Price $_{i, t-1}$ are the average spread and price. Results remain qualitatively similar for $2 \mathrm{~h}$ measurement interval. No.tickers is the number of tickers used in estimation. For SDAX stocks, we excluded the ticker HBH3 (HORNBACH HOLD.VZO O.N) that had only 15 trades on daily average. ***, ** and * denote either coefficient estimates that are significantly different from zero or test statistics that are significant at the $1 \%, 5 \%$, and $10 \%$ levels, respectively. 
Table 17: The Effect of HPITs on Market Efficiency (Autocorrelation-based Measure)

\begin{tabular}{lllllll}
\hline \multicolumn{2}{c}{ DAX } & \multicolumn{2}{c}{ MDAX } & \multicolumn{2}{c}{ SDAX } \\
\hline HPIT $_{t-1}$ & $-0.10^{* *}$ & $-0.06^{*}$ & $-0.03^{*}$ & -0.02 & 0.02 & 0.02 \\
& $(-2.41)$ & $(-1.74)$ & $(-1.74)$ & $(-1.17)$ & $(1.07)$ & $(1.25)$ \\
Price $_{t-1}$ & $-0.019^{* *}$ & 0.002 & 0.006 & -0.001 & $2.66 \mathrm{E}-05$ & $0.004^{* *}$ \\
& $(-2.02)$ & $(0.80)$ & $(0.51)$ & $(-0.36)$ & $(0.026)$ & $(2.01)$ \\
Range $_{t-1}$ & -0.002 & $-0.005^{* * *}$ & $7.26 \mathrm{E}-05$ & 0.001 & -0.002 & $-3.0 \mathrm{E}-04$ \\
& $(-1.02)$ & $(-2.69)$ & $(0.02)$ & $(0.18)$ & $(-0.82)$ & $(-0.12)$ \\
Spread $_{t-1}$ & $-0.016^{* * *}$ & $-0.005^{* * *}$ & $-0.003^{* * *}$ & $-0.002^{* * *}$ & 0.001 & $0.001^{* *}$ \\
& $(-9.30)$ & $(-4.52)$ & $(-6.76)$ & $(-5.10)$ & $(1.41)$ & $(2.46)$ \\
Constant & & $0.122^{* * *}$ & & $0.129^{* * *}$ & & $0.092^{* * *}$ \\
& & $(12.80)$ & & $(15.61)$ & & $(14.96)$ \\
\hline Effet & Fixed & Random & Fixed & Random & Fixed & Random \\
Observation & 7484 & 7484 & 12476 & 12476 & 6990 & 6990 \\
Adjusted $R^{2}$ & 0.032 & 0.011 & 0.011 & 0.007 & 0.001 & 0.006 \\
No.tickers & 30 & 30 & 50 & 50 & 49 & 49 \\
\hline
\end{tabular}

The table presents the fix- and random-effect panel regression results on variance-ratio based market price efficiency:

$$
\begin{aligned}
& |\rho|_{i, t}=\alpha_{i}+\gamma_{t}+\beta_{1} \times H P I T_{i, t-1}+\beta_{2} \times \log \left(\text { Price }_{i, t-1}\right)+\beta_{3} \times \text { Range }_{i, t-1}+\beta_{4} \times \text { Spread }_{i, t-1}+\epsilon_{i, t}, \\
& |\rho|_{i, t}=\mu+\alpha_{i}+\gamma_{t}+\beta_{1} \times H P I T_{i, t-1}+\beta_{2} \times \log \left(\text { Price }_{i, t-1}\right)+\beta_{3} \times \text { Range }_{i, t-1}+\beta_{4} \times \text { Spread }_{i, t-1}+\epsilon_{i, t},
\end{aligned},
$$

for DAX, MDAX, and SDAX stocks with $4 \mathrm{~h}$ measurement interval. HPIT $T_{i, t-1}$ is the the proportion of HPITs for stock $i$ during the period $i-1$, Range $e_{i, t-1}$ relates to the range between maximum and minimum price, and Spread $_{i, t-1}$ and Price $_{i, t-1}$ are the average spread and price. Results remain qualitatively similar for $2 \mathrm{~h}$ measurement interval. No.tickers is the number of tickers used in estimation. For SDAX stocks, we excluded the ticker HBH3 (HORNBACH HOLD.VZO O.N) that had only 15 trades on daily average. $* * *, * *$ and $*$ denote either coefficient estimates that are significantly different from zero or test statistics that are significant at the $1 \%, 5 \%$, and $10 \%$ levels, respectively. 


\section{References}

Admati, A.R., Pfleiderer, P., 1988. A theory of intraday patterns: Volume and price variability. Review of Financial Studies 1, 3-40.

Alexander, G.J., Peterson, M.A., 2007. An analysis of trade-size clustering and its relation to stealth trading. Journal of Financial Economics 84, 435 - 471.

Ascioglu, A., Comerton-Forde, C., McInish, T.H., 2011. Stealth trading: The case of the Tokyo stock exchange. Pacific-Basin Finance Journal 19, $194-207$.

Avramov, D., Chordia, T., Goyal, A., 2006. The impact of trades on daily volatility. Review of Financial Studies 19, 1241-1277.

Ball, C.A., Torous, W.N., Tschoegl, A.E., 1985. The degree of price resolution: The case of the gold market. Journal of Futures Markets 5, 29-43.

Banerjee, S., Davis, J., Gondhi, N., 2018. When transparency improves, must prices reflect fundamentals better? Review of Financial Studies 31, 2377-2414.

Barclay, M.J., Warner, J.B., 1993. Stealth trading and volatility. Journal of Financial Economics $34,281-305$.

Barron, O.E., Kim, O., Lim, S.C., Stevens, D.E., 1998. Using analysts' forecasts to measure properties of analysts' information environment. Accounting Review 73, 421-433.

Barron, O.E., Stuerke, P.S., 1998. Dispersion in analysts' earnings forecasts as a measure of uncertainty. Journal of Accounting, Auditing \& Finance 13, 245-270.

Bilodeau, Y., 2013. Xetraparser [computer software]. HEC Montréal .

Blasco, N., Corredor, P., 2017. The information environment, informed trading, and volatility. Journal of Behavioral Finance 18, 202-218.

Boehmer, E., Kelley, E.K., 2009. Institutional investors and the informational efficiency of prices. Review of Financial Studies 22, 3563-3594.

Cai, B.M., Cai, C.X., Keasey, K., 2006. Which trades move prices in emerging markets?: Evidence from china's stock market. Pacific-Basin Finance Journal 14, 453 - 466.

Campbell, J.Y., Grossman, S.J., Wang, J., 1993. Trading volume and serial correlation in stock returns. Quarterly Journal of Economics 108, 905-939. 
Cespa, G., Vives, X., 2015. The beauty contest and short-term trading. Journal of Finance 70, 2099-2154.

Chaboud, A., Chiquoine, B., Hjalmarsson, E., Clara, V., 2014. Rise of the machines: Algorithmic trading in the foreign exchange market. Journal of Finance 69, 2045-2084.

Chakravarty, S., 2001. Stealth-trading: Which traders' trades move stock prices? Journal of Financial Economics 61, $289-307$.

Collin-Dufresne, P., Fos, V., 2015. Do prices reveal the presence of informed trading? Journal of Finance 70, 1555-1582. doi:10.1111/jofi.12260.

Colombo, L., Femminis, G., Pavan, A., 2014. Information acquisition and welfare. Review of Economic Studies 81, 1438-1483.

Conrad, J., Wahal, S., Xiang, J., 2015. High-frequency quoting, trading, and the efficiency of prices. Journal of Financial Economics 116, 271-291.

Coval, J., Stafford, E., 2007. Asset fire sales (and purchases) in equity markets. Journal of Financial Economics 86, $479-512$.

Diamond, D.W., 1985. Optimal release of information by firms. Journal of Finance 40, 1071-1094.

Dufour, A., Engle, R.F., 2000. Time and the price impact of a trade. Journal of Finance 55, 2467-2498.

Dugast, J., Foucault, T., 2018. Data abundance and asset price informativeness. Journal of Financial Economics 130, 367-391.

Foster, F.D., Viswanathan, S., 1990. A theory of the interday variations in volume, variance, and trading costs in securities markets. Review of Financial Studies 3, 593-624.

Friedman, M., 1953. The case for flexible exchange rates. in Milton Friedman (ed), Essays in Positive Economics, University of Chicago Press, Chicago, IL .

Gao, P., Liang, P., 2013. Informational feedback, adverse selection, and optimal disclosure policy. Journal of Accounting Research 51, 1133-1158.

Garvey, R., Wu, F., 2014. Clustering of intraday order-sizes by uninformed versus informed traders. Journal of Banking and Finance 41, 222 - 235.

Goldstein, I., Yang, L., 2015. Information diversity and complementarities in trading and information acquisition. Journal of Finance 70, 1723-1765.

Gu, F., Wang, W., 2005. Intangible assets, information complexity, and analysts' earnings forecasts. Journal of Business Finance \& Accounting 32, 1673-1702. 
Han, B., Tang, Y., Yang, L., 2016. Public information and uninformed trading: Implications for market liquidity and price efficiency. Journal of Economic Theory 163, $604-643$.

Harris, L., 1991. Stock price clustering and discreteness. Review of Financial Studies 4, 389-415.

Hayek, F.A., 1945. The use of knowledge in society. American Economic Review 35, 519-530.

Hellwig, M.F., 1980. On the aggregation of information in competitive markets. Journal of Economic Theory 22, $477-498$.

Henderson, B.J., Pearson, N.D., Wang, L., 2014. New evidence on the financialization of commodity markets. Review of Financial Studies 28, 1285-1311.

Hodrick, L., P.C., M., 2005. Clustering in US stock prices after decimalization. Unpublished working paper, Rice University. .

Ikenberry, D., Weston, J., 2003. Liquidityg. Unpublished working paper, Columbia University .

Johnson, T.C., 2004. Forecast dispersion and the cross section of expected returns. Journal of Finance 59, 1957-1978.

Korajczyk, R.A., Murphy, D., 2018. High-frequency market making to large institutional trades. Review of Financial Studies 32, 1034-1067.

Kyle, A.S., 1985. Continuous auctions and insider trading. Econometrica 53, 1315-1335.

Lai, S., Ng, L., Zhang, B., 2014. Does PIN affect equity prices around the world? Journal of Financial Economics 114, $178-195$.

Lo, A.W., MacKinlay, A.C., 1988. Stock market prices do not follow random walks: Evidence from a simple specification test. Review of Financial Studies 1, 41-66.

Lo, A.W., MacKinlay, A.C., 1989. The size and power of the variance ratio test in finite samples: A Monte Carlo investigation. Journal of Econometrics 40, 203-238.

Menkhoff, L., Schmeling, M., 2010. Whose trades convey information? Evidence from a crosssection of traders. Journal of Financial Markets 13, $101-128$.

Milgrom, P., Stokey, N., 1982. Information, trade and common knowledge. Journal of Economic Theory $26,17-27$.

Moulton, P.C., 2005. You can't always get what you want: Trade-size clustering and quantity choice in liquidity. Journal of Financial Economics 78, 89- 119.

Niederhoffer, V., Osborne, M.F.M., 1966. Market making and reversal on the stock exchange. Journal of the American Statistical Association 61, 897-916. 
O'Hara, M., Yao, C., Ye, M., 2014. What's not there: Odd lots and market data. Journal of Finance 69, 2199-2236.

Rajan, R., Servaes, H., 1997. Analyst following of initial public offerings. Journal of Finance 52, 507-529.

Rosch, D.M., Subrahmanyam, A., van Dijk, M.A., 2016. The dynamics of market efficiency. Review of Financial Studies 30, 1151-1187.

Skjeltorp, J.A., Sojli, E., Tham, W.W., 2016. Flashes of trading intent at NASDAQ. Journal of Financial and Quantitative Analysis 51, 165-196.

Van Kervel, V., Menkveld, A., 2019. High-frequency trading around large institutional orders. Journal of Finance 74, 1091-1137.

Wang, J., 1993. A model of intertemporal asset prices under asymmetric information. Review of Economic Studies 60, 249-282.

Wyckoff, 1963. The psychology of stock market timing. Prentice-Hall, Englewood Cliffs, NJ . 